\title{
Nuclear Dbf2-related protein kinases (NDRs) in isolated cardiac myocytes and the myocardium: activation by cellular stresses and by phosphoprotein serine-/threonine-phosphatase inhibitors
}

Article

Accepted Version

Fuller, S. J., Pikkarainen, S., Tham, E. L., Cullingford, T. E., Molkentin, J. D., Cornils, H., Hergovitch, A., Hemmings, B. A., Clerk, A. and Sugden, P. H. (2008) Nuclear Dbf2-related protein kinases (NDRs) in isolated cardiac myocytes and the myocardium: activation by cellular stresses and by phosphoprotein serine-/threonine-phosphatase inhibitors. Cellular Signalling, 20 (8). pp. 1564-1577. ISSN 0898-6568 doi: https://doi.org/10.1016/j.cellsig.2008.04.013 Available at https://centaur.reading.ac.uk/18418/

It is advisable to refer to the publisher's version if you intend to cite from the work. See Guidance on citing.

To link to this article DOI: http://dx.doi.org/10.1016/j.cellsig.2008.04.013

Publisher: Elsevier

All outputs in CentAUR are protected by Intellectual Property Rights law, 
including copyright law. Copyright and IPR is retained by the creators or other copyright holders. Terms and conditions for use of this material are defined in the End User Agreement.

\section{www.reading.ac.uk/centaur}

\section{CentAUR}

Central Archive at the University of Reading

Reading's research outputs online 


\section{Nuclear Dbf2-related protein kinases (NDRs) in isolated cardiac}

\section{myocytes and the myocardium: Activation by cellular stresses and}

\section{by phosphoprotein serine-/threonine-phosphatase inhibitors}

Stephen J. Fuller ${ }^{\mathrm{a}}$ Sampsa Pikkarainen ${ }^{\mathrm{a}}$, El Li Tham ${ }^{\mathrm{a}}$, Timothy E. Cullingford ${ }^{\mathrm{a}}$, Jeffery D. Molkentin ${ }^{\mathrm{b}}$, Hauke Cornils ${ }^{\mathrm{c}}$, Alexander Hergovich ${ }^{\mathrm{c}}$, Brian A. Hemmings $^{\mathrm{c}}$, Angela Clerk ${ }^{\mathrm{a}}$, and Peter H Sugden ${ }^{\mathrm{a} *}$

${ }^{\mathrm{a}}$ National Heart and Lung Institute (NHLI) Division, Faculty of Medicine, Imperial College London, Flowers Building, Armstrong Road, London SW7 2AZ, UK

${ }^{\mathrm{b}}$ Department of Pediatrics, University of Cincinnati, Cincinnati Children's Hospital Medical Center, 3333 Burnet Ave., Cincinnati, Ohio 45229-3039, USA

${ }^{\mathrm{c}}$ Friedrich Miescher Institute for Biomedical Research, Maulbeerstrasse 66, CH 4002 Basel, Switzerland

Running title: Nuclear Dbf2-related protein kinases in the heart

* Corresponding author. NHLI Division, Faculty of Medicine, Imperial College London, Flowers Building (4th Floor), Armstrong Rd., London SW7 2AZ, UK. Tel.: +44 207594 3410; fax: +44 2075943419 .

E-mail address: p.sugden@imperial.ac.uk (P.H. Sugden). 


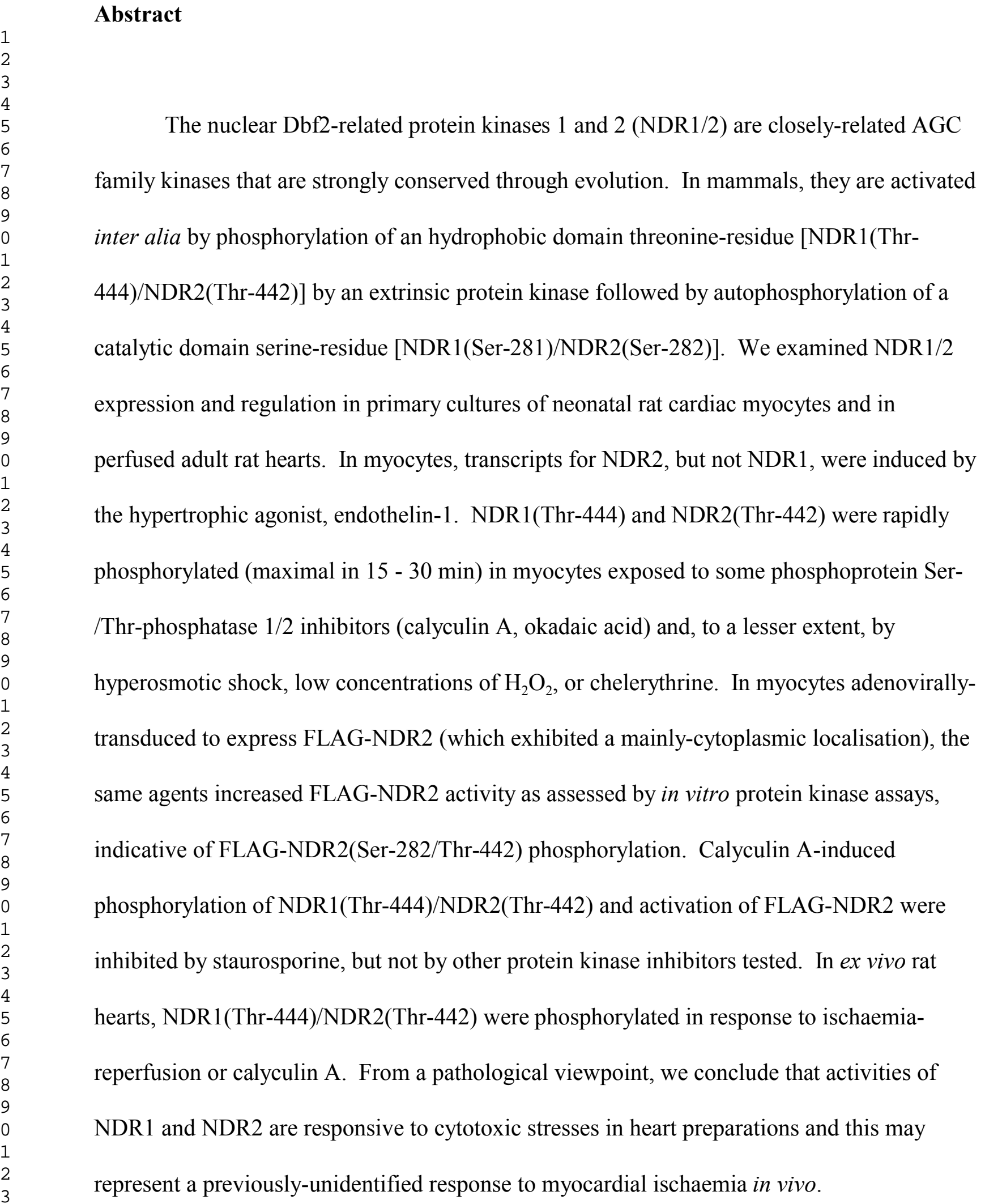


Keywords: Intracellular signalling; Protein phosphorylation; Nuclear Dbf2-related protein kinases (NDRs); Heart; Phosphoprotein Ser-/Thr-phosphatase inhibitors; Cellular stresses; Ischaemia and reperfusion injury 


\section{Introduction}

The nuclear Dbf2-related protein kinases (NDRs) are members of the AGC protein serine-/threonine-kinase family and are strongly conserved between species as diverse as yeasts and Homo sapiens [1,2]. Human (and mouse) cells express two isoforms encoded by separate genes, namely NDR1 (or serine-/threonine kinase 38, STK38) and NDR2 (or serine/threonine kinase 38-like, STK38L) [1,2]. These cells also express two NDR1-/NDR2related protein kinases, large tumour suppressor-1 (LATS1) and LATS2 [1,2]. The NDRs are widely-expressed in mouse [3] and human [4,5] tissues. In mouse, NDR1 transcripts are most-highly expressed in spleen and are also moderately expressed in brain, lung, thymus, testis and adipose tissue whereas NDR2 transcripts are most-highly expressed in the gastrointestinal tract (large and small intestine, stomach) and moderately expressed in testis [3]. The heart also expresses NDR1 and NDR2 transcripts, albeit at lower levels [3]. In human tissues, NDR1 and NDR2 are most highly expressed in thymus [4]. NDR1 is also highly expressed in human skeletal muscles whereas NDR2 is highly expressed in heart and brain [4]. It is not known whether these apparent differences in expression profiles between mouse and Homo sapiens are real or reflect inter-laboratory variations. The consensus sequence phosphorylated by this group of kinases has been variously reported to be (K/R)XX(S/T) (human NDR1 [6]), RXXS (preferred to RXXT) (yeast Dbf2 [7]) or $\mathrm{HX}(\mathrm{R} / \mathrm{H} / \mathrm{K}) \mathrm{XX}(\mathrm{S} / \mathrm{T})$ (human LATS1 [8]). The physiological functions of the NDRs in mammals are obscure. NDR2 may be involved in the organisation of the actin cytoskeleton in pheochromocytoma 12 (PC12) cells and its transcript is also rapidly but transiently induced in mouse amygdalae following Pavlovian fear conditioning training [9]. Recently, 
NDR1/NDR2 have been implicated in centrosome duplication with overexpression inducing centrosome overduplication [10].

In their inactive state, NDRs are autoinhibited by an insert between the catalytic domain subdomains VII and VIII (the autoinhibitory sequence) that is rich in basic residues [11] and was originally thought to represent a nuclear localisation signal [5]. Activation of NDRs involves regulatory phosphorylations and/or interactions with other proteins. As with many AGC kinases, NDRs are phosphorylated on two conserved residues, one in the Cterminal hydrophobic domain (Thr-444 in human, mouse or rat NDR1, Thr-442 in human or mouse NDR2) and one in the activation loop (or T loop) of the catalytic domain (Ser-281 in human, mouse or rat NDR1, Ser-282 in human or mouse NDR2) $[3,12]$. One protein kinase which phosphorylates NDR1(Thr-444)/NDR2(Thr-442) is the mammalian serine-/threonineSTE20-like kinase 3 (MST3) [13] with dephosphorylation catalysed by phosphoprotein serine-/threonine-phosphatase (PPP) PP2A $[3,12]$, now known as PPP2 . In contrast (and unusually for AGC kinases), phosphorylation of NDR1(Ser-281)/NDR2(Ser-282) is thought to represent an autophosphorylation $[3,13,14]$. In addition, activation may involve interactions with other proteins. Initially, the $\mathrm{S} 100 \mathrm{Ca}^{2+}$ binding protein was thought to be of major importance and to account for the $\mathrm{Ca}^{2+}$-sensitivity of NDR activation $[3,6,14]$. One suggestion is that phosphorylation of NDR2(Thr-75) in a S100/Ca ${ }^{2+}$-dependent manner leads to phosphorylation of NDR2(Thr-442) in the phospho-NDR2(Thr-75) [NDR2(PThr-75)] species by an unknown kinase $[14,15]$. The NDR2(Ser-281) residue in the NDR2(PThr$75 / P$ Thr-442) species is then autophosphorylated leading to activation $[14,15]$. However, the emphasis has now switched to the Mps-one binder (MOB) proteins which interact with basic residues in the N-terminal regulatory domains of the NDRs $[4,11]$. It is not entirely clear how 
MOB proteins activate NDRs. One view is that they bring NDRs to the plasma membrane for interaction with the upstream kinases for the hydrophobic domain phosphorylation, thus allowing enhanced autophosphorylation of the activation loop [16]. However, other mechanisms proposed are the release of the inhibition imposed by the autoinhibitory sequence [11] or, in yeast, perturbation and disruption of inhibitory self-associations [17].

Our interests in NDRs were stimulated by the observation that a microarray screen of the changes in transcript abundances following exposure of neonatal rat cardiac (ventricular) myocytes to the Gq protein-coupled receptor agonist, endothelin-1 (ET-1), revealed that NDR2 transcript abundances were increased $>2$-fold after $4 \mathrm{~h}$ [18]. In these cells, which become terminally differentiated during the perinatal period and are thus unable to undergo complete cycles of cell division, ET-1 is a powerful stimulator of hypertrophic growth, and this response has pathophysiological relevance [19-21]. These effects of ET-1 are brought about by rapid (within minutes) but transient activation of intracellular signalling pathways, particularly the extracellular signal-regulated kinase 1/2 (ERK1/2) cascade [22,23], and rapid but phasic activation of successive waves of gene expression, the earliest of which involve expression of immediate early genes [18]. Many of the early changes in gene expression are sensitive to inhibition of the ERK1/2 cascade $[18,24]$. Ultimately, these early changes lead to expression of genes associated with the established hypertrophic response [25]. Here, we have characterised the acute activation of NDRs in cardiac myocytes and have found that, whilst the expression of NDR2 was stimulated by ET-1, their activities were stimulated by cytotoxic interventions and particularly by PPP inhibitors. 


\section{Materials and methods}

\subsection{Materials}

With the exception of ET-1 (which was from Bachem.), biochemical reagents were from Sigma-Aldrich, Calbiochem or Alexis/Axxora, and laboratory chemicals were from VWR. Anti-NDR antibodies were raised to residues 59-86 of Homo sapiens NDR1 (for the measurement of the total pool) [16], and to a phosphopeptide (residues 436-450) encompassing NDR1(PThr-444) for measurement of the species phosphorylated in the hydrophobic domain [14]. These antibodies also recognise human NDR2 and NDR2(PThr442), respectively [13]. Since rat NDR1 is identical to human NDR1 and rat NDR2 is identical to human NDR2 in these immunogen regions, (see Supplementary Material, Fig. 1), these antibodies should and do recognise rat NDR1 and rat NDR2. Rabbit polyclonal antiOctA $\left(\mathrm{FLAG}^{\circledR}\right)$ Probe (D-8) (sc-807) and mouse monoclonal anti-OctA (FLAG $\left.{ }^{\circledR}\right)$ Probe (H6) (sc-7787) were from Santa Cruz Biotechnology. Secondary antibodies and fluorescent mounting medium were from Dako. $\left[\gamma-{ }^{32} \mathrm{P}\right] \mathrm{ATP}$, rainbow molecular weight markers, streptavidin-Texas red and Hyperfilm MP were from GE Healthcare.

\subsection{Primary culture of neonatal rat cardiac myocytes}

Myocytes were dissociated from the ventricles of 1- to 3-day-old Sprague-Dawley rat hearts by an adaptation of the method of Iwaki et al. [26] as previously described [27]. Unless stated otherwise, cells were plated in gelatin-coated Primaria culture dishes (BD Biosciences) at a density of $2 \times 10^{6}$ cells $/ 35 \mathrm{~mm}$ dish or $4 \times 10^{6}$ cells $/ 60 \mathrm{~mm}$ for $18 \mathrm{~h}$ in $15 \%$ 
(v/v) foetal calf serum. Unless experiments involved adenoviral infection, serum was withdrawn for the $24 \mathrm{~h}$ before experimentation.

\subsection{Infections with adenoviral vectors}

Details of the construction of the adenoviral vectors (Adv) encoding rat N-terminally FLAG-tagged wild-type NDR2 (FLAG-NDR2) and N-terminally FLAG-tagged NDR2PIFtide \{rat NDR2(1-432), i.e. lacking the hydrophobic domain residues 433-464, with the human PIFtide [3-phosphoinositide-dependent kinase 1 (PDK1) interacting fragment] from protein kinase N2 (PKN2, residues 969-983) spliced to the C-terminus, FLAG-NDR2PIFtide $\}$ are provided in the 'Supplementary Material' section. For Adv infections other than those for immunofluorescence staining, serum was withdrawn from myocytes $\left(2 \times 10^{6}\right.$ cells/35 mm dish) for approximately $6 \mathrm{~h}$ before infection with Adv encoding FLAG-NDR2 or FLAG-NDR2-PIFtide . In initial experiments, the multiplicities of infection were titrated so that the relative levels of expression of FLAG-NDR2 and endogenous NDR1/NDR2 were similar, as assessed by immunoblotting using the rabbit polyclonal anti-NDR1/NDR2 antibody. Likewise, the multiplicity of infection of Adv.FLAG-NDR2-PIFtide was adjusted so that expression of FLAG-NDR2-PIFtide and FLAG-NDR2 was approximately equal as shown using the rabbit polyclonal anti-FLAG antibody. Myocytes were used for experimentation (no change of medium) at $20 \mathrm{~h}$ after they had been infected. 


\subsection{Sodium dodecyl sulphate-polyacrylamide gel electrophoresis (SDS-PAGE) and} immunoblotting

Following exposure to agonists, myocytes were washed twice with ice-cold phosphate-buffered saline (PBS). For whole cell extracts, myocytes were scraped into 150 $\mu 1 /$ dish of Buffer A [20 mM $\beta$-glycerophosphate $\mathrm{pH}$ 7.5, 2 mM EDTA, 5 mM dithiothreitol (DTT), $50 \mathrm{mM} \mathrm{NaF}, 0.2 \mathrm{mM} \mathrm{Na}_{3} \mathrm{VO}_{4}, 2 \mu \mathrm{M}$ microcystin LR, $10 \mathrm{mM}$ benzamidine, $300 \mu \mathrm{M}$ phenylmethylsulphonyl fluoride (PMSF), $200 \mu \mathrm{M}$ leupeptin, $10 \mu \mathrm{M}$ trans-epoxy-succinyl-1-leucylamido-(4-guanidino)-butane (E64), containing 1\% (v/v) Triton $\mathrm{X}-100]$. Samples were incubated on ice for $10 \mathrm{~min}$ and then centrifuged $(10,000 \times \mathrm{g}, 5 \mathrm{~min}$, $\left.4^{\circ} \mathrm{C}\right)$. The supernatants were retained and protein contents measured by the Bradford assay (BioRad) [28]. Samples were boiled with 0.33 vol of SDS-PAGE sample buffer [10\% SDS (w/v), 13\% glycerol (v/v), 300 mM Tris-HCl pH 6.8, 130 mM DTT, 0.2\% bromophenol blue (w/v)]. Proteins (20 - 50 $\mu \mathrm{g} /$ lane, but equal for each blot) were separated by SDS-PAGE using 10\% (w/v) resolving gels and 4\% (w/v) stacking gels, and transferred to nitrocellulose as described previously [29]. Non-specific binding sites were blocked with 5\% (w/v) non-fat milk powder in TBST [20 mM Tris-HCl pH 7.5, $137 \mathrm{mM} \mathrm{NaCl,} \mathrm{0.1 \%} \mathrm{(v/v)} \mathrm{Tween} \mathrm{20].}$ Membranes were incubated (overnight, $4^{\circ} \mathrm{C}$ ) with primary antibodies $[1: 300$ for total NDR1/NDR2 and for NDR1(PThr-444)NDR2(PThr-442)] diluted in TBST containing 5\% (w/v) bovine serum albumin. The NDR1(PThr-444)NDR2(PThr-442) antibody in TBST/albumin could be used repeatedly providing it was stored at $4^{\circ} \mathrm{C}$ in the interim. Membranes were washed in TBST, incubated (60 min, room temperature) with horseradish peroxidase-conjugated secondary antibodies $(1 / 5,000)$ in TBST containing $1 \%(\mathrm{w} / \mathrm{v})$ nonfat milk powder, and were then washed in TBST. Bands were detected by enhanced 
chemiluminescence (Santa Cruz Biotechnology) using Hyperfilm MP and were quantified by scanning densitometry.

\subsection{Assay of FLAG-NDR2 activity (immunoprecipitation kinase assays)}

Myocytes were washed twice with ice-cold PBS and extracted into $100 \mu 1$ of Buffer B [20 mM Tris- $\mathrm{HCl} \mathrm{pH}$ 7.5, 10\% (v/v) glycerol, $100 \mathrm{mM} \mathrm{KCl,} 5 \mathrm{mM} \mathrm{MgCl}_{2}, 1 \mathrm{mM}$ EDTA, $0.05 \%$ (v/v) 2-mercaptoethanol, $5 \mathrm{mM}$ DTT, $5 \mathrm{mM} \mathrm{NaF}, 0.2 \mathrm{mM} \mathrm{Na}_{3} \mathrm{VO}_{4}, 2 \mu \mathrm{M}$ microcystin LR, $300 \mu \mathrm{M}$ PMSF, $200 \mu \mathrm{M}$ leupeptin, $10 \mu \mathrm{M}$ E64, containing 1\% (v/v) Triton X-100]. Samples were incubated on ice for $10 \mathrm{~min}$ and then centrifuged $\left(10,000 \times \mathrm{g}, 5 \mathrm{~min}, 4^{\circ} \mathrm{C}\right)$. The supernatants were retained and samples were taken for immunoprecipitation ( 75 or $90 \%$ of the supernatant used), protein determination by Bradford assay [28] and occasionally for immunoblotting. For immunoprecipitation, samples were added to Eppendorf tubes containing $20 \mu 1$ of protein A-Sepharose (1:1 slurry in Buffer B) and $5 \mu 1$ of rabbit anti-FLAG antibody that had been pre-incubated together overnight at $4^{\circ} \mathrm{C}$ with rotation. Samples were rotated overnight at $4^{\circ} \mathrm{C}$ and the pellets collected by centrifugation $\left(10,000 \times \mathrm{g}, 1 \mathrm{~min}, 4^{\circ} \mathrm{C}\right)$. The pellets were washed three times in Buffer B $(0.7 \mathrm{ml})$, once with kinase assay buffer (150 $\mu 1,20$ mM Tris-HCl pH 7.5, 1 mM DTT, $1 \mu \mathrm{M}$ microcystin LR, $4 \mu \mathrm{M}$ leupeptin, $1 \mathrm{mM}$ benzamidine) and finally resuspended in $45 \mu$ l of kinase assay buffer containing $1 \mathrm{mM}$ KKRNRRLSVA peptide substrate [6]. The recovery of FLAG-NDR2 or FLAG-NDR2PIFtide (estimated by immunoblotting using the monoclonal anti-FLAG antibody following immunoprecipitation with the rabbit polyclonal anti-FLAG antibody) was about $75 \%$ of the input. 
FLAG-NDR2 or FLAG-NDR2-PIFtide assays were initiated by the addition of $5 \mu 1$ of $1 \mathrm{mM}\left[\gamma^{-32} \mathrm{P}\right] \mathrm{ATP}(1 \mu \mathrm{Ci} /$ assay $) / 100 \mathrm{mM} \mathrm{MgCl}$ followed by incubation at $30^{\circ} \mathrm{C}$ for $30 \mathrm{~min}$. Reactions were terminated by the addition of $5 \mu 10.5 \mathrm{M}$ EDTA, $\mathrm{pH} 7.5$, followed by cooling on ice. Samples were centrifuged $\left(10,000 \times \mathrm{g}, 1 \mathrm{~min}, 4^{\circ} \mathrm{C}\right)$ and duplicate samples $(20 \mu \mathrm{l})$ of the supernatants were spotted onto P81 paper squares which were then washed in $75 \mathrm{mM}$ $\mathrm{H}_{3} \mathrm{PO}_{4}(4 \times 15 \mathrm{~min})$ on a rocking platform. Radioactivity was measured by Čerenkov radiation counting. Blanks (one with kinase assay buffer $+\left[\gamma-{ }^{32} \mathrm{P}\right] \mathrm{ATP} / \mathrm{MgCl}_{2}$ and one involving assay of KKRNRRLSVA kinase activity with immunopreciptates from cells infected with 'empty’ FLAG-tag Adv) were routinely included.

\subsection{Ex vivo heart preparations}

Hearts of female rats ( $279 \pm 6 \mathrm{~g}$ body wt.) that had littered 7-10 days previously were perfused retrogradely at $10 \mathrm{kPa}$ with Krebs-Henseleit bicarbonate-buffered saline as described previously [30]. In each set of experiments, following a 15 min retrograde stabilisation perfusion, hearts were either (i) perfused retrogradely for a further $20 \mathrm{~min}$, (ii) rendered globally-ischemic for $10 \mathrm{~min}$ by occlusion of the aortic inflow, (iii) reperfused retrogradely for $10 \mathrm{~min}$ following $10 \mathrm{~min}$ of global ischaemia, or (iv) perfused retrogradely with $40 \mathrm{nM}$ calyculin A for $5 \mathrm{~min}$. The hearts were then frozen between tongs cooled in liquid $\mathrm{N}_{2}$ and powdered by grinding in a pestle and mortar cooled in liquid $\mathrm{N}_{2}$. 


\subsection{Immunofluorescence staining}

Myocytes were cultured on glass coverslips [precoated with laminin $(20 \mu \mathrm{g} / \mathrm{ml}$ in PBS) and $1 \%(\mathrm{w} / \mathrm{v})$ gelatin] placed in $35 \mathrm{~mm}$ Primaria culture dishes at a density of $1.5 \times 10^{6}$ cells/dish for $18 \mathrm{~h}$ in the presence of $15 \%(\mathrm{v} / \mathrm{v})$ foetal calf serum. Myocytes were infected with Adv.FLAG-NDR2 (as described under 'Infection with adenoviral vectors' above) for 16 $\mathrm{h}$ in serum-free medium. The medium was discarded and the cells were washed with ice-cold PBS $(3 \times 0.5 \mathrm{ml})$. Cells were fixed in $4 \%(\mathrm{w} / \mathrm{v})$ formaldehyde $(10 \mathrm{~min})$, permeabilised with $0.3 \%(\mathrm{v} / \mathrm{v})$ Triton X-100 in PBS, and non-specific antibody binding was blocked with $10 \%$ $(\mathrm{w} / \mathrm{v})$ horse serum in PBS containing 0.3\% (v/v) Triton X-100. FLAG-NDR2 was detected by incubation of cells with mouse anti-FLAG antibody ( $1: 25$ dilution, $\left.2 \mathrm{~h}, 37^{\circ} \mathrm{C}\right)$, a biotinylated goat anti-mouse IgG secondary antibody (1:200 dilution, 30 min, room temperature) and streptavidin-Texas Red (1:200 dilution, $30 \mathrm{~min}$, room temperature). Sarcomeric structures were identified by FITC-phalloidin (1:100, $1 \mathrm{~h}$, room temperature). Nuclei were stained with Hoechst $33258(50 \mu \mathrm{g} / \mathrm{ml}, 10 \mathrm{~min}$, room temperature). Cells were washed with PBS $(3 \times 0.5 \mathrm{ml})$ between each incubation. Slides were mounted and examined using a Zeiss Axioskop microscope with a 100× oil immersion objective and photographed using a digital camera.

\subsection{Quantitative PCR ( $q P C R)$}

RNA was extracted and cDNA synthesised using reverse transcription as previously described [31]. qPCR was performed using a Real-Time PCR System model 7500 (Applied Biosystems). Amplifications were carried out in optical 96-well reaction-plates (Applied Biosystems) with each well containing $12.5 \mu 1$ of SYBR Green Jump Start Taq Readymix 
(Sigma Aldrich Chemical Co.), $5 \mu 1$ of oligonucleotide primers (5 pmol each of forward and reverse primers) and $7.5 \mu 1$ of cDNA template (diluted 15 -fold in water). Details of the primers used are given in Table 1. qPCR analysis of Gapdh was performed as a control and the relative quantification protocol was used. PCR conditions for all primer pairs were $50^{\circ} \mathrm{C}$ for $2 \mathrm{~min}, 95^{\circ} \mathrm{C}$ for $10 \mathrm{~min}$ (Jump-Start Taq polymerase activation step), followed by 40 cycles of $95^{\circ} \mathrm{C}$ for $15 \mathrm{~s}$ and $59^{\circ} \mathrm{C}$ for $60 \mathrm{~s}$. Following qPCR, dissociation curve analysis was routinely performed to check for aberrant amplification products (e.g. primer-dimers).

\subsection{Calculation of results and statistical methods}

Within a single experiment (i.e. a single preparation of cardiac myocytes), results are expressed relative to the maximum signal for the quantified immunoblots or FLAG-NDR2 activities. Statistical significance was tested using two-tailed Student's $t$ test for paired samples or one-way ANOVA with Tukey's multiple comparison test as appropriate. A statistically significant difference required that $P<0.05$. 


\section{Results}

\subsection{Molecular cloning of rat NDR2 cDNA}

In an Affymetrix rat 2302.0 array screen of primary cultures of neonatal rat cardiac myocytes exposed to ET-1 for 4 h, two probe-sets (1372955_at and 1383299_at) identified an expressed sequence tag (EST) that was significantly upregulated to 2.28-fold (average of both probe-sets) of the zero-time control [18]. A BLAST search revealed that this rat EST corresponded to the mouse NDR2 nucleotide sequence. Rat NDR2 cDNA was cloned as described in 'Supplementary Material' and the sequence obtained (bases 1 - 2406) deposited in Genbank (accession no. EF444939.1). The translational open reading frame (bases 151 1542, 464 amino acids) was aligned against human, mouse and rat NDR1, and human and mouse NDR2 using the ClustalW program (www.ebi.ac.uk/clustalw) (Supplementary Material, Fig. 1). The primary structure of rat NDR2 (calculated molecular mass: $53.6 \mathrm{kDa}$ ) is identical to mouse NDR2 except for replacement of mouse Asn-15 and Val-216 with Ser15 and Ile-216, respectively, in rat NDR2.

Using qPCR, we verified that NDR2 mRNA was significantly upregulated (3- to 4fold of zero-time control) by ET-1 at $1.5 \mathrm{~h}$ and remained upregulated at this level until at least $8 \mathrm{~h}$ (Fig. 1A). In contrast, NDR1 mRNA was not significantly altered over the same time period (Fig. 1B). However, using the antibody which recognises both NDR1 and NDR2, we were unable to detect any change in the abundances of NDR proteins by ET-1 over the same time period (results not shown). The primary structures of NDR1 and NDR2 show a high degree of identity (Supplementary Material, Fig. 1) and the proteins are very similar in molecular mass (NDR1 is $54.0 \mathrm{kDa}$ ), so that it is possible that any newly-synthesised NDR2 would not necessarily be readily detectable against the pre-existing NDR1/NDR2 background. 


\subsection{Phosphorylation of NDR1(Thr-444)/NDR2(Thr-442) and activation of FLAG-NDR2 by calyculin A}

Calyculin A is an inhibitor of PPP1 and PPP2 (formerly known as PP2A), inhibiting each approximately equipotently (Table 2$)$. Calyculin A (200 nM) induced a rapid phosphorylation (detectable by $1 \mathrm{~min}$ ) of NDR1(Thr-444)/NDR2(Thr-442) in cardiac myocytes, reaching a maximum at $10-20$ min and remaining stable for at least 90 min (Fig. 2A). There was no change in total NDR1/NDR2 abundance (Fig. 2A). Because of their similarities in molecular mass, NDR1 and NDR2 usually co-migrate though, if samples are electrophoresed for longer, occasionally two closely-migrating bands are detectable with the antibody to NDR1(PThr-444)/NDR2(PThr-442). Using this anti-phospho-NDR antibody, the $\mathrm{EC}_{50}$ for the calyculin A-mediated phosphorylation of NDR1(Thr-444)/NDR2(Thr-442) was computed to be approximately $10 \mathrm{nM}$ (Fig. 2B). The activation of NDR1 or NDR2 requires dual phosphorylation of NDR1(Ser-281/Thr-444) or NDR2(Ser-282/Thr-442), the phosphorylation of NDR1(Ser-281)/NDR2(Ser-282) representing autophosphorylations following the NDR1(Thr-444)/NDR2(Thr-442) phosphorylations [3,13,14]. However, the available antibody [14] raised against NDR1(PSer-281)/NDR2(PSer-282) is of relatively low affinity/efficacy and we could not examine the phosphorylation of these sites. We did investigate whether, following adenovirally-mediated transfer of FLAG-NDR2, the activity of FLAG-NDR2 as measured by immunoprecipitation kinase assays was commensurate with phosphorylation of NDR1(Thr-444)/NDR2(Thr-442). We adjusted the adenoviral infections so that we achieved essentially equal expression of FLAG-NDR2 and endogenous NDR1/NDR2 (Fig. 2C). Immunprecipitation kinase assays using the anti-FLAG antibody showed that, as for the phosphorylation of NDR1(Thr-444)/NDR2(Thr-442) (Fig. 2B), the activity of FLAG-NDR2 was also stimulated by calyculin A with an $\mathrm{EC}_{50}$ of approximately $10 \mathrm{nM}$ (Fig. 2D). We noticed that calyculin A also appeared to be positively chronotropic in these myocyte cultures and thus considered the possibility that contractile activity might be responsible for NDR phosphorylation. However, contractile arrest by blockade of the L-type- 
and T-type $\mathrm{Ca}^{2+}$ channels with $10 \mu \mathrm{M}$ nifedipine and $1.8 \mu \mathrm{M}$ mibefradil did not reduce the effects of calyculin A (results not shown).

\subsection{Phosphorylation of NDR1(Thr-444)/NDR2(Thr-442) by hyperosmotic shock and oxidative stress}

Exposure of myocytes to hyperosmotic shock ( $0.5 \mathrm{M}$ sorbitol) resulted in a rapid (maximal in 10 min or less) phosphorylation of NDR1(Thr-444)/NDR2(Thr-442) (Fig. 3A) though, unlike phosphorylation elicited by $200 \mathrm{nM}$ calyculin A (Fig. 2A), phosphorylation had declined by $90 \mathrm{~min}$. Oxidative stress $\left(0.5 \mathrm{mM} \mathrm{H}_{2} \mathrm{O}_{2}\right)$ caused a somewhat slower phosphorylation (maximal at about $20 \mathrm{~min}$ ) and this again declined by $90 \mathrm{~min}$ (Fig. 3B). In neither condition was there any change in the abundance of total NDR1/NDR2 (Fig. 3A-B). The phosphorylation elicited by oxidative stress was detectable at $0.1 \mathrm{mM} \mathrm{H}_{2} \mathrm{O}_{2}$ and showed a distinct peak occurring at about $0.5 \mathrm{mM}$ (Fig. 3C). At higher concentrations ( $1-10 \mathrm{mM}$ $\mathrm{H}_{2} \mathrm{O}_{2}$ ), phosphorylation declined. There were no losses of myocytes from the dishes at up to $3 \mathrm{mM} \mathrm{H}_{2} \mathrm{O}_{2}$, as shown by the immunoblots against total NDR1/NDR2 (Fig. 3C), though it is possible that some losses occurred at $10 \mathrm{mM} \mathrm{H}_{2} \mathrm{O}_{2}$.

\subsection{Relative potencies of phosphorylation of NDR1(Thr-444)/NDR2(Thr-442) and activation of FLAG-NDR2 by calyculin A, hyperosmotic shock and $\mathrm{H}_{2} \mathrm{O}_{2}$, and identification of additional modulators}

We examined the ability of a wide range of known modulators of myocardial processes to mediate phosphorylation of NDR1(Thr-444)/NDR2(Thr-442) and/or activation of FLAG-NDR2 (see Supplementary Material, Table 2 for details of agonists/interventions without effect). We also set any modulators identified as being effective in a hierarchy in comparison with calyculin A. Chelerythrine is allegedly a protein kinase $\mathrm{C}$ (PKC) inhibitor [32], though this has been questioned at least in the case of the $\mathrm{PKC} \alpha$ isoform [33]. In 
cardiac myocytes, chelerythrine causes conventional and novel PKC-independent pyknosis, comparison with $200 \mathrm{nM}$ calyculin A (NDR1/NDR2 control not subtracted), chelerythrine is about 45 to $50 \%$ as effective in inducing phosphorylation of NDR1(Thr-444)/NDR2(Thr-442) (Fig. 4A), but is only about 20\% (control FLAG-NDR2 activity not subtracted) as effective in inducing activation of FLAG-NDR2 (Fig. 4B). Likewise, $0.5 \mathrm{mM} \mathrm{H}_{2} \mathrm{O}_{2}$ is about 40 to $45 \%$ as effective as $200 \mathrm{nM}$ calyculin A in inducing phosphorylation of NDR1(Thr-444)/NDR2(Thr442 ) but is only about 5 to $10 \%$ as effective in inducing activation of FLAG-NDR2 (Fig. 4AB). The PPP2 inhibitor okadaic acid (OKA), which also inhibits PPP1 though less effectively (Table 2), is known to induce phosphorylation and activation of NDR1/NDR2 [3,12]. In our hands, after a $60 \mathrm{~min}$ incubation, $1 \mu \mathrm{M}$ OKA is almost as effective as incubation with 200 $\mathrm{nM}$ calyculin A for 20 or $60 \mathrm{~min}$ in inducing phosphorylation of NDR1(Thr-444)/NDR2(Thr442) (Fig. 4A). However, following a 20 min incubation, $1 \mu \mathrm{M}$ OKA does not elicit any statistically significant phosphorylation of NDR1(Thr-444)/NDR2(Thr-442) compared with a control incubation (Fig. 4A), though there is a statistically-insignificant increase to about $30 \%$ of the level observed with $200 \mathrm{nM}$ calyculin A for 20 or $60 \mathrm{~min}$. For activation of FLAGNDR2, OKA (1 $\mu \mathrm{M}, 20 \mathrm{~min})$ was $<10 \%$ as effective as calyculin A (200 nM, $20 \mathrm{~min})$ (Fig. 4C). However, after a 60 min incubation with OKA, this had increased to 45 to 50\% (Fig. 4C). The general conclusions are (i) calyculin A is the most powerful stimulator of NDR1(Thr-444)/NDR2(Thr-442) and activation of FLAG-NDR2 that we have identified, (ii) generally, activation of FLAG-NDR2 lags behind phosphorylation of NDR1(Thr444)/NDR2(Thr-442). Because it could be argued that this difference was related to the different species of NDRs examined (endogenous NDR1/NDR2 or FLAG-NDR2), we examined the phosphorylation of endogenous NDR1/NDR2 and FLAG-NDR2 in FLAGNDR2-transduced cardiac myocytes (Fig. 4D). This was feasible because of the lower mobility of FLAG-NDR2. No differences between the phosphorylation of endogenous NDR1(Thr-444)/NDR2(Thr-442) and FLAG-NDR2(Thr-442) could be identified for any agonist. We also calculated the approximate ratio of expression of FLAG-NDR2(PThr-442) 
to endogenous NDR1(PThr-444)/NDR2(PThr-442) using the anti-NDR1(PThr444)/NDR2(PThr-442) antibody. If it can be assumed that the most significant factor influencing this ratio is the levels of expression, the value should approximate to the FLAGNDR2/endogenous NDR1/NDR2 ratio (Fig. 2C). The FLAG-NDR2(PThr-442)/endogenous NDR1(PThr-444)/NDR2(PThr-442) ratio is approximately 1.5, i.e. similar to that observed in Fig. 2C.

Given that calyculin A is an equipotent PPP1/PPP2 inhibitor whereas OKA inhibits PPP2 more potently (Table 2), the spectrum of NDR1/NDR2 phosphorylation (Fig. 4A,D) and FLAG-NDR2 activation (Fig. 4C) suggests that the effects of calyculin A could be exerted primarily through PPP1. We therefore investigated the effects of tautomycetin (200 $\mathrm{nM}$ for up to $4 \mathrm{~h}$ ) which is primarily a PPP1 inhibitor (Table 2) and fostriecin (200 $\mathrm{nM}$ for up to $4 \mathrm{~h}$ ) which is primarily a PPP2 inhibitor (Table 2) on activity of FLAG-NDR2. Neither inhibitor had any detectable effect (results not shown). Furthermore, the potent PPP1/PPP2 inhibitor (Table 2), microcystin LR $(2 \mu \mathrm{M})$, did not activate FLAG-NDR2, nor did it induce phosphorylation of endogenous NDR1(Thr-444)/NDR2(Thr-442) or FLAG-NDR2(Thr-442). The effects of PPP inhibitors on the NDRs are therefore limited largely to calyculin A, with lesser effects of OKA.

\subsection{Inhibitors of calyculin A-mediated activation of FLAG-NDR2}

We investigated the ability of a number of known protein kinase inhibitors to reduce the activation of FLAG-NDR2 resulting from exposure of cardiac myocytes to $50 \mathrm{nM}$ calyculin A. Of the inhibitors examined, the only inhibitor with any effect was staurosporine. Staurosporine was originally identified as a PKC inhibitor [35] but is now recognised to be a non-selective protein kinase inhibitor [36] (see also Reference [33] for 7hydroxystaurosporine). Staurosporine exhibited an $\mathrm{IC}_{50}$ of approximately $40 \mathrm{nM}$ for the inhibition of the activation of FLAG-NDR2 by $50 \mathrm{nM}$ calyculin A (Fig. 5A). This inhibition 
was not attributable to 'carry-over' of staurosporine from the cell incubations into the assay because addition of staurosporine up to $1 \mu \mathrm{M}$ for the last 5 min of a 20 min exposure of myocytes to $50 \mathrm{nM}$ calyculin A [i.e. after FLAG-NDR2 would have been essentially completely activated by calyculin A (Fig. 2A) but prior to extraction and immunoprecipitation] did not result in any significant reduction in FLAG-NDR2 activity. It is possible that staurosporine may not inhibit phosphorylation of NDR1(Thr-444)/NDR2(Thr442) but could selectively inhibit the autophosphorylation of NDR1(Ser-281)/NDR2(Ser282). We therefore also examined its effects on the calyculin A-stimulated phosphorylation of NDR1(Thr-444)/NDR2(Thr-442) and FLAG-NDR2(Thr-442) (Fig. 5B). Staurosporine also inhibited the calyculin A-stimulated phosphorylation of endogenous NDR1(Thr444)/NDR2(Thr-442) and of exogenous FLAG-NDR2(Thr-442) (Fig. 5B), and this in itself should prevent phosphorylation of FLAG-NDR2(Ser-282) and its activation (Fig. 5A). We do not know whether staurosporine also inhibits the autophosphorylation of NDR1(PThr444)/NDR2(PThr-442) at the T loop residues.

The following were ineffective in inhibiting activation of FLAG-NDR2 by calyculin A: $100 \mu \mathrm{M}$ genistein (a generalised Tyr-protein kinase inhibitor [37]), $10 \mu \mathrm{M}$ GF109203X (generalised PKC inhibitor [38] which also inhibits a number of other protein kinases [33]), $20 \mu \mathrm{M}$ HA1077 (a PKN inhibitor [39] which inhibits a number of protein kinases including protein kinase A and Rho kinase [33]), $10 \mu \mathrm{M}$ SB203580 [inhibitor of the $\alpha$ and $\beta$ isoforms of p38-mitogen-activated protein kinase (p38-MAPK) [40]] or $10 \mu \mathrm{M}$ Y27632 (Rho kinase inhibitor [41] which also inhibits PKN [33]). Equally, no inhibitor activated FLAG-NDR2 in the absence of calyculin A (results not shown). The phosphoinositide 3-kinase (PI3K) inhibitor, LY294002 [42] (50 $\mu \mathrm{M})$, did not inhibit NDR1(Thr-444)/NDR2(Thr-442) phosphorylation or activation of FLAG-NDR2 (results not shown). [We ensured that the LY294002 preparation used was active by demonstrating that it prevented stimulation of protein kinase B/Akt (PKB/Akt) phosphorylation by $300 \mathrm{nM}$ insulin.] 


\subsection{Activity of FLAG-NDR2-PIFtide}

Mutation of the two phosphorylation sites in the catalytic and hydrophobic domains of NDR1 (i.e. Ser-281 and Thr-444) to acidic residues is relatively-ineffective in producing a kinase with increased constitutive activity [3]. A constitutively-activated species can be produced by splicing NDR2(1-432) to PKN2(969-983) to produce NDR2-PIFtide, as has been previously described for human NDR2 [3]. We examined the activities of FLAG-NDR2 (rat) and FLAG-NDR2-PIFtide (rat-human chimera) in the absence or presence of calyculin A and approximately matching the abundances of FLAG-NDR2 and FLAG-NDR2-PIFtide (Fig. 6). After subtracting the activity seen following infection with the adenoviral blank (which was not accurately matched for multiplicity of infection with Adv.FLAG-NDR2 and Adv.FLAGNDR2-PIFtide) and correcting for differences in relative abundance, the constitutive activity of FLAG-NDR2-PIFtide was found to be approximately 10-fold greater than that of FLAGNDR2 (Fig. 6). Not unexpectedly, FLAG-NDR2-PIFtide (which lacks the Thr-442 phosphorylation site) could not be activated by calyculin A (Fig. 6).

\subsection{Intracellular localisation of NDR2 in cardiac myocytes}

Using the antibodies available, we were unsuccessful in staining for endogenous dephospho-NDR (as were Hergovich et al. [16] in U2-OS cells), nor were we successful in staining for phospho-NDRs. We therefore examined the intracellular localisation of NDR2 by infecting myocytes with Adv.FLAG-NDR2 and staining for the FLAG epitope. FLAGNDR2 localised to the cytoplasm in cardiac myocytes and was clearly excluded from the nucleus (Fig. 7). The pattern of staining was somewhat uneven in the cytoplasm and there was a suggestion of some partition of NDR2 to the plasma membrane. There was no evidence of any change in localisation following exposure to $30 \mathrm{nM}$ calyculin A for $20 \mathrm{~min}$ (results not shown). 
3.8. Phosphorylation of NDR1(Thr-444)/NDR2(Thr-442) in intact heart by calyculin A or by ischaemia and reperfusion

Calyculin A proved to be the most powerful stimulator of NDR1(Thr444)/NDR2(Thr-442) phosphorylation and activator of FLAG-NDR2 in neonatal rat cardiac myocytes that we identified here (see Fig. 4A-D). Some of the interventions resulting in phosphorylation of NDR1(Thr-444)/NDR2(Thr-442) and activation of FLAG-NDR2 in myocytes are established cytotoxic stresses in neonatal cardiac myocytes (chelerythrine [34], $\mathrm{H}_{2} \mathrm{O}_{2}$ [43] and hyperosmotic shock [44]). However, it is important to show that these findings are not confined to neonatal cells. We therefore investigated the effects of calyculin A and of pathologically-relevant cardiac stresses, namely ischaemia and ischaemiareperfusion in intact adult rat hearts. As expected, calyculin A (40 nM, $5 \mathrm{~min})$ induced a highly-significant $(\mathrm{P}<0.0001)$ increase in the phosphorylation of NDR1(Thr-444)/NDR2(Thr442) in perfused hearts (Fig. 8). Although there was some suggestion that global ischaemia (10 min) increases phosphorylation of NDR1(Thr-444)/NDR2(Thr-442), this was not significantly different from control normoxic perfusions. In contrast, global ischaemia (10 min) followed by reperfusion $(10 \mathrm{~min})$ resulted in significant $(P<0.0001)$ phosphorylation of NDR1(Thr-444)/NDR2(Thr-442) which, after subtraction of the control, was equivalent to about $30 \%$ of the level induced by $40 \mathrm{nM}$ calyculin A for 5 min (Fig. 8). 


\section{Discussion}

\subsection{General comments}

The NDRs are highly-conserved protein Ser-/Thr-kinases that are members of the AGC kinase superfamily $[1,2]$. In terms of their activation, the current scheme is that NDR1(Thr-444) and NDR2(Thr-442) (the hydrophobic domain residues) are first phosphorylated by an upstream kinase and then NDR1(PThr-444) and NDR2 $(P T h r-442)$ each autophosphorylate their own $\mathrm{T}$ loop residues in their catalytic domains to produce the active species NDR1(PSer-281/PThr-444) and NDR2(PSer-282/PThr-442) $[1,2]$. The fact that activation of FLAG-NDR2 tends to lag behind phosphorylation of NDR1(Thr444)/NDR2(Thr-442) (for example, seen clearly for OKA in Fig. 4A, C) suggests that this ordered phosphorylation occurs in cardiac myocytes. The agonists that lead to activation of NDRs are poorly-characterised and their biological functions in higher organisms are poorlyunderstood. Given that NDR1/NDR2 are highly-conserved through evolution, it seems likely that they possess important functions. NDR2 may be involved in organisation of the actin cytoskeleton [9] and, recently, a role for NDR1/NDR2 in centrosome duplication has been identified $[2,10]$. Although populations of myocytes range from the mononucleate to the multinucleate and thus must possess some capacity for karyokinesis, they are generally considered to be terminally-differentiated and incapable of cytokinesis. It therefore seems unlikely that centrosome duplication is of major importance in their case.

\subsection{Expression of the NDR1 and NDR2 genes}

At the level of mRNA, NDR2 transcripts are rapidly but transiently induced in amygdalae of mice following emotional stress [9] and, here, we show that NDR2 mRNA is also rapidly induced by ET-1 in neonatal rat cardiac myocytes (Fig. 1A). In contrast, NDR1 mRNA is not induced by ET-1 (Fig. 1B). Presumably, their promoter regions are sufficiently 
different to allow differential transcription or else transcript stability may be involved. Using microarrays (Affymetrix rat 2302.0 array at 30 min or the more-restricted Affymetrix rat U34A microarrays at 2 or $4 \mathrm{~h}$ ) in conjunction with semi-quantitative PCR and/or qPCR, we have shown that, for ET-1, the activation of the ERK1/2 cascade is required for the increased expression of the majority of transcripts upregulated at $30 \mathrm{~min}, 2 \mathrm{~h}$ or $4 \mathrm{~h}[18,24]$. We have not yet studied in detail whether this is the case for NDR2 mRNA [the U34A microarrays did not have a probe set for rat NDR2 [24] and we only studied the 30 min time point in our more recent study [18] when NDR2 transcripts are not upregulated (Fig. 1A)].

\subsection{Involvement of PPPs in NDR phosphorylation and activation}

The PPP superfamily, of which there are seven sub-families, is encoded by 20 genes in Homo sapiens and these encode about 28 catalytic subunits [45]. There is also a multiplicity of regulatory subunits (particularly for PPP1) which are involved in targeting (to control subcellular localisation and substrate specificity) and regulation (e.g. inhibitory subunits). Previously, OKA has been identified as an agent that stimulates phosphorylation and activation of NDR1/NDR2 [3,12]. This is presumably achieved by inhibiting a PPP, allowing unknown NDR1/NDR2 kinases to phosphorylate NDRs. Here, we show that, at maximally-effective concentrations, calyculin A is more effective than OKA in inducing NDR1(Thr-444)/NDR2(Thr-442) phosphorylation and activation of FLAG-NDR2 in neonatal rat cardiac myocytes after 20 min (Fig. 4A, C). Although calyculin A or OKA treatment result in equivalent NDR1(Thr-444)/NDR2(Thr-442) phosphorylation after 60 min, activation of FLAG-NDR2 with OKA is still less than with calyculin A (Fig. 4A, C). A similar hysteresis between phosphorylation of NDR1(Thr-444)/NDR2(Thr-442) and activation of FLAG-NDR2 is seen in the individual comparisons of all interventions (Fig. 4). It probably reflects the ordered nature of the phosphorylation of first NDR1(Thr-444)/NDR2(Thr-442) followed by NDR1(Ser281)/NDR2(Ser-282). In previous work in which COS cells were transfected with tagged NDRs, OKA ( $1 \mu \mathrm{M}, 60 \mathrm{~min})$ stimulated NDR activity by 12 - to 25 - 
fold [3,12]. This compares with 21-fold in our analogous experiments (Fig. 4C), but we find that calyculin A (200 nM, 60 min) stimulates activity by about 45-fold (Fig. 4C). We do not know whether these differences result from trivial causes (e.g. differences in permeability) or whether there are mechanistic differences. Importantly, calyculin A stimulates phosphorylation of NDR1(Thr-444)/NDR2(Thr-442) in adult perfused heart (Fig. 8), confirming that the findings in neonatal myocyte are not confined this particular stage of maturation.

Though calyculin A and OKA may show differing selectivities for PPP1 and PPP2 [OKA has a 50-fold greater $\mathrm{IC}_{50}$ for PPP1 than PPP2 (Table 2)], they are both relatively potent inhibitors of both PPPs at least in vitro (Table 2). We had hoped possibly to discriminate between PPP1 and PPP2 using the more selective inhibitors fostriecin and tautomycetin (Table 2), and we also studied the effects of microcystin LR. However, we were never able to activate FLAG-NDR2 with these, nor did microcystin LR cause phosphorylation of endogenous NDR1(Thr-444)/NDR2(Thr-442) or FLAG-NDR2(Thr-442). We do not understand the reasons for this. They could be trivial (e.g. permeability), but all ineffective inhibitors have been used in intact cell preparations previously. Equally, there may be PPP1/PPP2 holoenzyme species which are insensitive to fostriecin, tautomycetin or microcystin LR, or there may be PPP species other than PPP1 and PPP2 which are sensitive to calyculin A and OKA but which are not sensitive to fostriecin, tautomycetin or microcystin LR. Furthermore, it may be incorrect to assume that the actions of calyculin A or OKA are exerted at the level of the PPP(s) directly responsible for deposphorylating NDRs. The point(s) of action could lie further upstream. The current predominating view that PPP2 is responsible for the dephosphorylation of NDR1(PSer-281/PThr-444) and NDR2(PSer282/PThr-442) is based on experiments in intact cells with OKA [3,12], and on in vitro experiments involving dephosphorylation of NDR1(PSer-281/PThr-444) with recombinant PPP2 [12]. However, the concentration of OKA used in these experiments was $1 \mu \mathrm{M}$ or 300times greater than the $\mathrm{IC}_{50}$ even for PPP1. Whilst there may be unassessed permeability 
problems related to the access of OKA to the intracellular compartment, these experiments are not unequivocal. Equally, in vitro experiments with recombinant PPP2 are not unequivocal because of the possibility that any PPP might dephosphorylate the phosphoNDRs if present in high enough excess. The PPP(s) responsible for dephosphorylating NDR1(PSer-281/PThr-444) and NDR2(PSer-282/PThr-442) remain somewhat obscure.

\subsection{Phosphorylation and activation of NDR1/NDR2}

Here, we have shown that NDR1/NDR2 are phosphorylated and activated by a variety of stimuli in cardiac myocytes and intact heart. Though we have not identified the kinases responsible, these experiments show that the endogenous upstream signalling is intact. With respect to the established kinases, PDK1 is often involved in phosphorylation of a T loop Ser/Thr-residue in AGC kinases, i.e. a Ser-/Thr-residue in the catalytic domain [46,47]. Activation of PDK1, which is probably potentially active in the basal state by virtue of its high affinity interactions with 3-phosphoinositides [48], is mediated by PI3K-dependent phosphorylation of phosphatidylinositol 4,5-bisphosphate $\left[\operatorname{PtdIns}(4,5) P_{2}\right]$ to $\operatorname{PtdIns}(3,4,5) P_{3}$ and lies downstream from the insulin or insulin-like growth factor 1 (IGF1) receptor protein tyrosine kinases (RPTKs) as well as other RPTKs [47,49]. These T loop phosphorylations are either regulatory (i.e. respond to external stimuli to change phosphorylation state and activity of the kinase involved) or are essentially facilitative (i.e. are constitutive rather than regulatory, but are necessary for activity). Evidence has been presented that neither PDK1 nor insulin or IGF1 is involved in the regulation of NDR1 [12] though, equally, contradictory evidence has been presented that IGF1-activated PDK1 is an NDR2(Thr-442) kinase and this phosphorylation precedes the autophosphorylation of the T loop residue [15]. We did not find any evidence that insulin (which does cause PI3K/PDK1-dependent phosphorylation of PKB/Akt in cardiac myocytes [50]) stimulated phosphorylation of NDR1(Thr444)/NDR2(Thr-442) (Supplementary Material, Table 2A) nor did LY294002 inhibit the phosphorylation of NDR1(Thr-444)/NDR2(Thr-442) or activation of FLAG-NDR2 by 
calyculin A. Our data thus do not support a role for PDK1. A second mode of activation NDR2(Thr-75), then phosphorylation of NDR2(Thr-442) in the NDR2(PThr-75) species by an unknown kinase, and finally autophosphorylation of NDR2(Ser-281) in the NDR2(PThr75/PThr-442) species leading to activation $[14,15]$. The necessary increase in $\mathrm{Ca}^{2+}$ concentrations is mediated through phospholipase $\mathrm{C} \gamma(\operatorname{PLC} \gamma)$ and hydrolysis of $\operatorname{PtdIns}(4,5) P_{2}$ to the $\mathrm{Ca}^{2+}$-mobilising 'second messenger', inositol 1,4,5-trisphosphate $\left[\operatorname{Ins}(1,4,5) P_{3}\right][15]$. However, in cardiac myocytes, platelet-derived growth factor stimulates $\operatorname{Ptd} \operatorname{Ins}(4,5) P_{2}$ hydrolysis and Tyr-phosphorylation of PLC $\gamma 1$ [50], but does not stimulate NDR1(Thr444)/NDR2(Thr-442) phosphorylation (Supplementary Material, Table 2A). Furthermore, ET-1 stimulates PLC $\beta$-mediated hydrolysis of $\operatorname{PtdIns}(4,5) P_{2}$ in cardiac myocytes [51] but does not stimulate phosphorylation of NDR1(Thr-444)/NDR2(Thr-442) or activation of FLAG-NDR2 (Supplementary Material, Table 2A and 2B).

One upstream kinase that may be involved in the phosphorylation of NDR1(Thr444)/NDR2(Thr-442) is mammalian STE20-like kinase 3 (MST3) [13], also known as STK24, a member of the germinal centre kinase family GCK-III subclass [52,53]. The regulation of these kinases in terms of the agonists that lead to their activation is not wellunderstood and indeed Pombo et al. [53] state that 'To date, no stimuli have been shown to activate MST3'. In fact, like the NDRs, MST3 is activated by OKA [13] and calyculin A [54]. Overexpression of MST3 in HEK293 cell causes fragmentation of DNA indicative of apoptosis/cell death [55]. If the NDRs are involved, this would suggest that their activation by MST3 should be pro-apoptotic. It will be of interest to examine whether, in addition to calyculin A and OKA, pro-apoptotic stimuli such as chelerythrine, hyperosmotic shock and $\mathrm{H}_{2} \mathrm{O}_{2}$ activate MST3. One characteristic of the upstream NDR1(Thr-444)/NDR2(Thr-442) kinase that we did identify is that it is inhibited by staurosporine (Fig. 5A-B). Staurosporine and its derivatives inhibit a broad spectrum of Ser-/Thr-protein kinases (including PDK1), though a number of kinases are relatively insensitive $[33,36]$. A prerequisite of the 
NDR1(Thr-444)/NDR2(Thr-442) kinase is that its activity or activation should be inhibited by staurosporine.

\subsection{Pathophysiological relevance of interventions which stimulate phosphorylation of NDR1(Thr-444)/NDR2(Thr-442) and activation of FLAG-NDR2}

The interventions which stimulate phosphorylation of NDR1(Thr-444)/NDR2(Thr442) and activation of FLAG-NDR2 most clearly are calyculin A and OKA (Fig. 2A-B, D, Fig. 8) and OKA (Fig. 4A, C). Other than possibly contributing to the understanding of diarrhetic shellfish poisoning for which OKA is responsible, these findings have very little pathophysiological significance. The phosphorylation of NDR1(Thr-444)/NDR2(Thr-442) and activation of FLAG-NDR2 are also stimulated by a relatively-limited subset of cell stress stimuli, notably hyperosmotic shock (Fig. 3A, Fig. 4A-B) and oxidative stress (Fig. 3B-C, Fig. 4A-B) in neonatal cardiac myocytes. Perhaps most notably, global ischaemia/reperfusion, a pathologically-important pro-apoptotic stress in the heart [56], stimulates phosphorylation of NDR1(Thr-444)/NDR2(Thr-442) in isolated adult rat hearts (Fig. 8). Although there is a suggestion that ischaemia alone also resulted in phosphorylation of NDR1(Thr-444)/NDR2(Thr-442), this result did not achieve statistical significance (Fig. 8). In isolated hearts, the stress-activated protein kinases of the MAPK family are activated (by phosphorylation) by ischaemia and ischaemia/reperfusion [57]. Thus, ischaemia activates p38-MAPKs and this is increased on reperfusion [56,57]. Ischaemia alone does not activate the c-Jun N-terminal kinases (JNKs), but they are activated on reperfusion [57]. However, p38-MAPKs and JNKs are activated by a wider range of cellular stresses (e.g. anisomycin) in cardiac myocytes than those that lead to phosphorylation of NDR1(Thr-444)/NDR2(Thr-442) (Supplementary Material, Table 2A) or activation of FLAG-NDR2 (Supplementary Material, Table 2B), and they are also activated by some $\mathrm{G}$ protein receptor agonists, e.g. ET-1 and phenylephrine [58-60]. As with phosphorylation of NDR1(Thr-444)/NDR2(Thr-442) and activation of FLAG-NDR2 (Fig. 3B-C, Fig. 4A-B), JNKs and p38-MAPKs are activated by 
oxidative stress in cardiac myocytes and the perfused heart [30,61], and our findings indicate

\subsection{Subcellular localisation of NDR2}

The subcellular localisation of the NDRs as assessed by either immunofluorescence or subcellular fractionation is controversial. NDR1 was first thought to be localised to the nucleus [5]. Tagged NDR1 overexpressed in HeLa cells also appears to localise primarily to the nucleus with evidence of an additional cytoplasmic localisation at higher levels of expression [4]. Somewhat surprisingly in view of the similarity in their primary sequences (see Supplementary Material, Fig. 1), endogenous or overexpressed tagged NDR2 appears to localise primarily to the cytoplasm (sometimes in a punctate manner) with evidence of nuclear localisation or perinuclear concentration when expression at higher levels $[3,4]$. However, Hergovich et al. [16] (see also Reference [10]) have now demonstrated that both endogenous NDR1 and overexpressed NDR1 are primarily cytoplasmic and suggest that some previous studies demonstrating a nuclear localisation resulted from misinterpretation of immunofluorescence data. In our hands, FLAG-NDR2 expressed ectopically to about the same level as total endogenous NDRs localises to the cytoplasm of cardiac myocytes, though there is some evidence of increased concentration at the plasma membrane (Fig. 7). This might perhaps be in keeping with the need for NDR2, on association with MOB proteins, to translocate to the plasma membrane for activation by phosphorylation $[11,16]$. We did not assess the localisation of NDR1. 


\subsection{NDR2-PIFtide}

When protein kinases are activated by Ser-/Thr- phosphorylation, the normal tactic to increase the level of constitutive activity is to mutate the Ser-/Thr- residues to Asp- or Glu-. However, mutation of NDR1(Ser-281) does not affect activity and mutation of NDR1(Thr444) caused only a 1.5 - to 2-fold increase in activity [12]. In PKB/Akt, phosphorylation of the homologous hydrophobic domain Ser- (Ser-472, Ser-473 or Ser-474) depending on the isoform $(\alpha, \beta$ or $\gamma)$ ] results in full activation of $\mathrm{PKB} / \mathrm{Akt}$ phosphorylated on the PDK1 site in the T loop (Thr-308 or Thr-309) [49]. Crystallographic studies show that phosphorylation of $\mathrm{PKB} \beta / \mathrm{Akt} 2$ (Ser-474) results in a disorder-to-order transition of the $\alpha \mathrm{C}$ helix and interaction with $\mathrm{PKB} / \mathrm{Akt}(P \mathrm{Thr}-309)$ [63]. This ordered structure can be mimicked by the hydrophobic motif C-terminal region of PKN2 which contains the so-called PIFtide sequence which is highly ordered [64]. In NDR2, replacement of residues 433-464 with the PKN2 PIF domain (human PKN2 residues 969-983) produces an NDR2 species which was estimated as possessing a 20-fold increase in activity over the wild type NDR2 and in which the T loop phosphorylation site is phosphorylated [3]. The specific activity of tagged NDR2-PIFtide is about 2-fold greater than that of the tagged NDR2(wild type) from COS cells treated with 1 $\mu \mathrm{M}$ OKA for $60 \mathrm{~min}$, and its activity might be slightly further increased by exposure of the cells to OKA [3]. We estimate here that the activity of FLAG-NDR2-PIF-tide was about 10fold greater than FLAG-NDR2(wild-type) (Fig. 6), and this was still much less (about 15$20 \%$ ) than that of FLAG-NDR2(wild-type) that had been activated by $200 \mathrm{nM}$ calyculin A for 20 min. Calyculin A did not further activate FLAG-NDR2-PIFtide (Fig. 6), presumably because Thr-442 is absent. We were hoping that ectopic expression of the activated FLAGNDR2-PIFtide might cause some obvious changes in its subcellular localisation and/or myocyte morphology. However, we were not able to detect any such changes and a moredetailed examination of any prospective phenotypes is currently being undertaken. 


\section{Acknowledgements}

This work was supported by Fondation Leducq 'Transatlantic Network of Excellence' Grant 05-CVD2 to P.H.S., A.C. and J.D.M., and by British Heart Foundation Grants RG/2001007 and PG/07/060/23276 to P.H.S. and A.C. S.P. was supported by Fellowships from the Sigrid Jusélius Foundation, the Finnish Foundation for Cardiovascular Research, the Instrumentarium Foundation, the Aarne and Aili Turunen Foundation, the Maud Kuistila Foundation and the Paavo Nurmi Foundation. A.H was supported by Oncosuisse Grant OCS 1942-08-2006. Research at the Friedrich Miescher Institute is supported by the Novartis Research Foundation. 


\section{References}

[1] A. Hergovich, M.R. Stegert, D. Schmitz, B.A. Hemmings, Nat. Rev. Mol. Cell Biol. 7 (2006) 253.

[2] A. Hergovich, H. Cornils, B.A. Hemmings, Biochim. Biophys. Acta 1784 (2008) 3.

[3] M.R. Stegert, R. Tamaskovic, S.J. Bichsel, A. Hergovich, B.A. Hemmings, J. Biol. Chem. 279 (2004) 23806.

[4] E. Devroe, H. Erdjument-Bromage, P. Tempst, P.A. Silver, J. Biol. Chem. 279 (2004) 24444.

[5] T. Millward, P. Cron, B.A. Hemmings, Proc. Natl. Acad. Sci. U. S. A. 92 (1995) 5022.

[6] T.A. Millward, C.W. Heizmann, B.W. Schäfer, B.A. Hemmings, EMBO J. 17 (1998) 5913.

[7] A.S. Mah, A.E. Elia, G. Devgan, J. Ptacek, M. Schutkowski, M. Snyder, M.B. Yaffe, R.J. Deshaies, BMC Biochem. 6 (2005) 22.

[8] Y. Hao, A. Chun, K. Cheung, B. Rashidi, X. Yang, J. Biol. Chem. 283 (2008) 5496.

[9] O. Stork, A. Zhdanov, A. Kudersky, T. Yoshikawa, K. Obata, H.-C. Pape, J. Biol. Chem. 279 (2004) 45773.

[10] A. Hergovich, S. Lamla, E.A. Nigg, B.A. Hemmings, Mol. Cell 25 (2007) 625.

[11] S.J. Bichsel, R. Tamaskovic, M.R. Stegert, B.A. Hemmings, J. Biol. Chem. 279 (2004) 35228.

[12] T.A. Millward, D. Hess, B.A. Hemmings, J. Biol. Chem. 274 (1999) 33847.

[13] M.R. Stegert, A. Hergovich, R. Tamaskovic, S.J. Bichsel, B.A. Hemmings, Mol. Cell. Biol. 25 (2005) 11019.

[14] R. Tamaskovic, S.J. Bichsel, H. Rogniaux, M.R. Stegert, B.A. Hemmings, J. Biol. Chem. 278 (2003) 6710.

[15] A. Suzuki, T. Ogura, H. Esumi, J. Biol. Chem. 281 (2006) 13915.

[16] A. Hergovich, S.J. Bichsel, B.A. Hemmings, Mol. Cell. Biol. 25 (2005) 8259.

[17] M.C. Hou, D.A. Guertin, D. McCollum, Mol. Cell. Biol. 24 (2004) 3262. 
[18] T.E. Cullingford, T. Markou, S.J. Fuller, A. Giraldo, S. Pikkarainen, G. Zoumpoulidou, A. Alsafi, C. Ekere, T.J. Kemp, J.L. Dennis, L. Game, P.H. Sugden, A. Clerk, Genome Biol. 9 (2008) R32[.

[19] H.E. Shubeita, P.M. McDonough, A.N. Harris, K.U. Knowlton, C.C. Glembotski, J.H. Brown, K.R. Chien, J. Biol. Chem. 265 (1990) 20555.

[20] H. Ito, Y. Hirata, M. Hiroe, M. Tsujino, S. Adachi, T. Takamoto, M. Nitta, K. Taniguchi, F. Marumo, Circ. Res. 69 (1991) 209.

[21] P.H. Sugden, J. Mol. Cell. Cardiol. 35 (2003) 871.

[22] M.A. Bogoyevitch, P.E. Glennon, P.H. Sugden, FEBS Lett. 317 (1993) 271.

[23] M.A. Bogoyevitch, P.E. Glennon, M.B. Andersson, A. Clerk, A. Lazou, C.J. Marshall, P.J. Parker, P.H. Sugden, J. Biol. Chem. 269 (1994) 1110.

[24] R.A. Kennedy, T.J. Kemp, P.H. Sugden, A. Clerk, J. Mol. Cell. Cardiol. 41 (2006) 236. [25] A. Clerk, T.E. Cullingford, S.J. Fuller, A. Giraldo, T. Markou, S. Pikkarainen, P.H. Sugden, J. Cell. Physiol. 212 (2007) 311.

[26] K. Iwaki, V.P. Sukhatme, H.E. Shubeita, K.R. Chien, J. Biol. Chem. 265 (1990) 13809.

[27] M.A. Bogoyevitch, A. Clerk, P.H. Sugden, Biochem. J. 309 (1995) 437.

[28] M.M. Bradford, Anal. Biochem. 72 (1976) 248.

[29] A. Clerk, M.A. Bogoyevitch, M.B. Andersson, P.H. Sugden, J. Biol. Chem. 269 (1994) 32848 .

[30] A. Clerk, S.J. Fuller, A. Michael, P.H. Sugden, J. Biol. Chem. 273 (1998) 7228.

[31] T.J. Kemp, H.C. Causton, A. Clerk, Biochem. Biophys. Res. Commun. 307 (2003) 416.

[32] J.M. Herbert, J.M. Augereau, J. Gleye, J.P. Maffrand, Biochem. Biophys. Res.

Commun. 172 (1990) 993.

[33] S.P. Davies, H. Reddy, M. Caivano, P. Cohen, Biochem. J. 351 (2000) 95.

[34] S. Yamamoto, K. Seta, C. Morisco, S.F. Vatner, J. Sadoshima, J. Mol. Cell. Cardiol. 33 (2001) 1829.

[35] T. Tamaoki, H. Nomoto, I. Takahashi, Y. Kato, M. Morimoto, F. Tomita, Biochem. Biophys. Res. Commun. 135 (1986) 397. 
[36] F. Meggio, A. Donella Deanna, M. Ruzzene, A.M. Brunati, L. Cesaro, B. Guerra, T. Meyer, H. Mett, D. Fabbro, P. Furet, G. Dobrowolska, L.A. Pinna, Eur. J. Biochem. 234 (1995) 317.

[37] T. Akiyama, J. Ishida, S. Nakagawa, H. Ogawara, S. Watanabe, N. Itoh, M. Shibuya, Y. Fukami, J. Biol. Chem. 262 (1987) 5592.

[38] D. Toullec, P. Pianetti, H. Coste, P. Bellevergue, T. Grand-Perret, M. Ajakane, V. Baudet, P. Boissin, E. Boursier, F. Loriolle, L. Duhamel, D. Charon, J. Kirilovsky, J. Biol. Chem. 266 (1991) 15771.

[39] N.E. Torbett, A. Casamassima, P.J. Parker, J. Biol. Chem. 278 (2003) 32344.

[40] S. Kumar, P.C. McDonnell, R.J. Gum, A.T. Hand, J.C. Lee, P.R. Young, Biochem. Biophys. Res. Commun. 235 (1997) 533.

[41] M. Uehata, S. Ishizaki, H. Satoh, T. Ono, T. Kawahara, T. Morishita, H. Tamakawa, K. Yamagami, J. Inui, M. Maekawa, S. Narumiya, Nature 389 (1997) 990.

[42] C.J. Vlahos, W Matter,F,, K.Y. Hui, R.F. Brown, J. Biol. Chem. 269 (1994) 5241.

[43] S.A. Cook, P.H. Sugden, A. Clerk, Circ. Res. 85 (1999) 940.

[44] M. Galvez, M.P. Morales, J.M. Eltit, P. Ocaranza, L. Carrasco, X. Campos, M.

Sapag-Hagar, G. Díaz-Araya, S. Lavandero, Cell Tissue Res. 304 (2001) 279.

[45] M. Gallego, D.M. Virshup, Curr. Opin. Cell Biol. 17 (2005) 197.

[46] A.C. Newton, Biochem. J. 370 (2003) 361.

[47] A. Mora, D. Komander, D.M.F. van Aalten, D.R. Alessi, Semin. Cell Dev. Biol. 15 (2004) 161.

[48] R.A. Currie, K.S. Walker, A. Gray, M. Deak, A. Casamayor, C.P. Downes, P. Cohen, D.R. Alessi, J. Lucocq, Biochem. J. 337 (1999) 575.

[49] B. Vanhaesebroeck, D.R. Alessi, Biochem. J. 346 (2000) 561.

[50] A. Clerk, I.-K.S. Aggeli, K. Stathopoulou, P.H. Sugden, Cell. Signal. 18 (2006) 225.

[51] A. Clerk, P.H. Sugden, J. Mol. Cell. Cardiol. 29 (1997) 1593.

[52] I. Dan, N.M. Watanabe, A. Kusumi, Trends Cell Biol. 11 (2001) 220. 
[53] C.M. Pombo, T. Force, J. Kyriakis, E. Nogueira, M. Fidalgo, J. Zalvide, Front. Biosci. $12(2007) 850$.

[54] K. Schinkmann, J. Blenis, J. Biol. Chem. 272 (1997) 28695.

[55] C.-F.Y. Huang, Y.-M. Wu, C.-Y. Hsu, W.-S. Lee, M.-D. Lai, T.-J. Lu, C.-L. Huang, T.-H. Leu, H.-M. Shih, H.-I. Fang, D.R. Robinson, H.-J. Kung, C.-J. Yuan, J. Biol. Chem. 277 (2002) 34367.

[56] X.L. Ma, S. Kumar, F. Gao, C.S. Louden, B.L. Lopez, T.A. Christopher, C. Wang, J.C. Lee, G.Z. Feuerstein, T.L. Yue, Circulation 99 (1999) 1685.

[57] M.A. Bogoyevitch, J. Gillespie-Brown, A.J. Ketterman, S.J. Fuller, R. Ben-Levy, A. Ashworth, C.J. Marshall, P.H. Sugden, Circ. Res. 79 (1996) 162.

[58] M.A. Bogoyevitch, A.J. Ketterman, P.H. Sugden, J. Biol. Chem. 270 (1995) 29710.

[59] A. Clerk, A. Michael, P.H. Sugden, Biochem. J. 333 (1998) 581.

[60] A. Lazou, P.H. Sugden, A. Clerk, Biochem. J. 332 (1998) 459.

[61] A. Clerk, A. Michael, P.H. Sugden, J. Cell Biol. 142 (1998) 523.

[62] G. Solaini, D.A. Harris, Biochem. J. 390 (2005) 377.

[63] J. Yang, P. Cron, V.M. Good, V. Thompson, B.A. Hemmings, D. Barford, Nat. Struct. Biol. 9 (2002) 940.

[64] J. Yang, P. Cron, V. Thomson, V.M. Good, D. Hess, B.A. Hemmings, D. Barford, Mol. Cell 9 (2002) 1227.

[65] M. Suganuma, H. Fujiki, S. Okabe, S. Nishiwaki, D. Brautigan, T.S. Ingebritsen, M.R. Rosner, Toxicon 30 (1992) 873.

[66] M. Roberge, C. Tudan, S.M.F. Hung, K.W. Harder, F.R. Jirik, H. Anderson, Cancer Res. 54 (1994) 6115.

[67] S. Mitsuhashi, N. Matsuura, M. Ubukata, H. Oikawa, H. Shima, K. Kikuchi, Biochem. Biophys. Res. Commun. 287 (2001) 328. 
Table 1.

Quantitative PCR primers. Nucleotide positions in transcripts are shown in parentheses for each primer. mRNA sequences for established genes

were obtained from the Rat Genome Database (http://rgb.mcw.edu, viewed at http://www.ncbi.nlm.nih.gov/entrez).

\begin{tabular}{lllll}
\hline Gene & Accession & Size & Forward primer & Reverse primer \\
& no./probe set & $(\mathrm{bp})$ & & \\
\hline NDR1 & NM_001015025 & 176 & TTCGAGGGCCTGACAGCCAG (807-826) & TCAGAGACTTGACTAGGACGCAGT (960-983) \\
NDR2 & EF444939.1 & 238 & AGGTCATCCGTTCTTTGAGGGTGTG (1284-1308) & ACGTGGGGATGGAGCCTCGCTG (1501-1522) \\
Gapdh & NM_017008 & 83 & GCTGGCATTGCTCTCAATGACA (1738-1759) & TCCACCACCCTGTTGCTGTA (1801-1820) \\
\hline
\end{tabular}


Table 2. Potencies of PPP1/PPP2 inhibitors.

1

2

3

4

5

6
Inhibitor

$\mathrm{IC}_{50}$ against

Reference

\begin{tabular}{lccc}
\hline & PPP1 & PPP2 & \\
\cline { 2 - 3 } Calyculin A & $0.3 \mathrm{nM}$ & $0.13 \mathrm{nM}$ & \\
Okadaic acid & $3.4 \mathrm{nM}$ & $0.07 \mathrm{nM}$ & {$[65]$} \\
Microcystin LR & $0.1 \mathrm{nM}$ & $0.1 \mathrm{nM}$ & {$[65]$} \\
Fostriecin & $4 \mu \mathrm{M}$ & $40 \mathrm{nM}$ & {$[66]$} \\
Tautomycetin & $1.6 \mathrm{nM}$ & $62 \mathrm{nM}$ & {$[67]$} \\
& & & \\
\hline
\end{tabular}




\section{Figure legends}

Fig. 1. Quantitative PCR of (A) NDR2 and (B) NDR1 mRNA abundances in cardiac myocytes exposed to endothelin-1 (ET-1). Myocytes (4 independent preparations for NDR2, 5 independent preparations for NDR1) were exposed to $100 \mathrm{nM}$ ET-1 for the times indicated and RNA extracted. Transcript levels were normalised to those of Gapdh, then expressed relative to the zero-time controls. Results are expressed as means \pm SEM. Statistical significance versus the zero-time control: *,$P<0.05$; **, $P<0.01$ by a paired two-tailed Student's $t$ test.

Fig. 2. Phosphorylation of NDR1(Thr-444) and NDR2(Thr-442), and activation of FLAGNDR2 in cardiac myocytes exposed to calyculin A. All results are expressed as means \pm SEM. (A) Myocytes (3 independent preparations) were exposed to $200 \mathrm{nM}$ calyculin A for the times indicated and phosphorylation of NDR1(Thr-444)/NDR2(Thr-442) assessed by immunoblotting with an anti-NDR phosphopeptide antibody. There were no changes in the abundances of total NDR1/NDR2. The intensities of the NDR1(PThr-444)/NDR2(PThr-442) signals were assessed by scanning densitometry and expressed relative to the maximum values which occurred after either 45 or 90 min of exposure of myocytes to calyculin A, depending on the time point at which the greater extent of phosphorylation was observed. (B) The dependence of NDR1(Thr-444)/NDR2(Thr-442) phosphorylation on calyculin A concentration was assessed in myocytes (4 independent preparations) exposed to calyculin A for 20 min. The intensities of the NDR1(PThr-444)/NDR2(PThr-442) signals were assessed as described under (A) and expressed relative to the maximum values which occurred at either 100 or $300 \mathrm{nM}$ calyculin A, depending on the concentration at which the greater extent of phosphorylation was observed. (C) Myocytes were infected with Adv.FLAG-NDR2. Immunoblotting with an anti-NDR1/NDR2 antibody showed that endogenous NDR1/NDR2 and FLAG-NDR2 were expressed in approximately equal amounts. (D) The dependence of FLAG-NDR2 activity on calyculin A concentrations was assessed in infected myocytes (4 
independent preparations) exposed to calyculin A for $20 \mathrm{~min}$. Activities were expressed relative to the maximum values which occurred at either 100 or $300 \mathrm{nM}$ calyculin $\mathrm{A}$, depending on the concentration at which the greater activity was observed.

Fig. 3. Phosphorylation of NDR1(Thr-444) and NDR2(Thr-442) in cardiac myocytes exposed to hyperosmotic shock or oxidative stress. All results are expressed as means \pm SEM. (A and B) Myocytes ( 3 independent preparations) were exposed to hyperosmotic shock (0.5 M sorbitol) (A) or $0.5 \mathrm{mM} \mathrm{H}_{2} \mathrm{O}_{2}$ (B) and phosphorylation of NDR1(Thr-444)/NDR2(Thr-442) assessed by immunoblotting with an anti-NDR phosphopeptide antibody. There were no changes in the abundances of total NDR1/NDR2. The intensities of the NDR1(PThr444)/NDR2(PThr-442) signals were assessed by scanning densitometry and were expressed relative to the maximum values which occurred after either 10 or 20 min of exposure of myocytes to hyperosmotic shock (A) or 20 or 45 min of exposure to $0.5 \mathrm{mM} \mathrm{H}_{2} \mathrm{O}_{2}$ (B), depending on the time point at which the greater extent of phosphorylation was observed. (C) The dependence of NDR1(Thr-444)/NDR2(Thr-442) phosphorylation on $\mathrm{H}_{2} \mathrm{O}_{2}$ concentration was assessed in myocytes ( 5 independent preparations) exposed to $\mathrm{H}_{2} \mathrm{O}_{2}$ for $20 \mathrm{~min}$. The intensities of the NDR1(PThr-444)/NDR2(PThr-442) signals were assessed as described under (A and B) and were expressed relative to the maximum values which occurred at 0.5 $\mathrm{mM} \mathrm{H}_{2} \mathrm{O}_{2}$.

Fig. 4. Direct comparison of induction of phosphorylation of NDR1(Thr-444) and NDR2(Thr-442), and activation of FLAG-NDR2 in cardiac myocytes exposed to a range of interventions. HOS, hyperosmotic shock; OKA, okadaic acid. (A) Myocytes (3 to 8 independent preparations) were exposed to NDR modulators at the concentrations and for the times indicated and phosphorylation of NDR1(Thr-444)/NDR2(Thr-442) assessed by immunoblotting with an anti-NDR phosphopeptide antibody. The intensities of the NDR1(PThr-444)/NDR2(PThr-442) signals were assessed by scanning densitometry. Results are expressed relative to maximum phosphorylation which was observed with $200 \mathrm{nM}$ 
calyculin $\mathrm{A}$ at either $20 \mathrm{~min}$ or $60 \mathrm{~min}$, depending on the time point at which the greater $P<0.05 ; * *, P<0.01, * * *, P<0.001$ versus control or $\uparrow, P<0.01$ versus $\mathrm{OKA}(1 \mu \mathrm{M}, 60 \mathrm{~min})$ by a 1-tailed ANOVA with Tukey's multiple comparison test. (B and C) FLAG-NDR2 activity following exposure of myocytes [8 independent preparations in (B), 3 independent preparations in $(\mathrm{C})$ ] to the interventions indicated and expressed relative to the maximum activity which was observed with $200 \mathrm{nM}$ calyculin A at either $20 \mathrm{~min}$ or $60 \mathrm{~min}$, depending on the time point at which the greater activity was observed. Results are means \pm SEM. (D) Myocytes expressing the FLAG-NDR2 transgene (2 independent preparations of myocytes) were exposed to NDR modulators at the concentrations and for the times indicated and phosphorylation of NDR1(Thr-444)/NDR2(Thr-442) assessed by immunoblotting with an anti-NDR phosphopeptide antibody. The intensities of the NDR1(PThr-444)/NDR2(PThr442) signals were assessed as under (A). The upper band represents FLAG-NDR2(PThr-442) and the two lower bands represent NDR1(PThr-444)/NDR2(PThr-442). The unfilled bars in the histogram represent FLAG-NDR2(PThr-442) whereas the filled bars represent endogenous NDR1(PThr-444)/NDR2(PThr-442). Results are expressed relative to maximum phosphorylation which was observed with $200 \mathrm{nM}$ calyculin A at $60 \mathrm{~min}$ and are means $\pm \mathrm{SD}$.

Fig. 5. Inhibition of calyculin A-induced activation of FLAG-NDR2 and phosphorylation of endogenous NDR1(Thr-444)/NDR2(Thr-442) and FLAG-NDR2(Thr-442) by staurosporine. Adv, adenoviral vector. (A and B) Myocytes were preincubated for 20 min with staurosporine at the concentrations indicated, then were exposed to $50 \mathrm{nM}$ calyculin A for a further $20 \mathrm{~min}$. (A) Inhibition of calyculin A-induced stimulation of FLAG-NDR2 activity by staurosporine. FLAG-NDR2 activity in control 1 was assessed at $50 \mathrm{nM}$ calyculin A (20 min) in the absence of staurosporine and results were expressed relative to this. FLAG-NDR2 activity in control 2 was assessed in the absence of $50 \mathrm{nM}$ calyculin A and staurosporine. (B) Inhibition of calyculin A-induced phosphorylation of endogenous NDR1(Thr-444)/NDR2(Thr-442) (left hand immunoblot panel and unfilled bars), and of endogenous NDR1(Thr-444)/NDR2(Thr- 
442) and FLAG-NDR2(Thr-442) (right hand immunoblot panel and filled bars) was assessed

by immunoblotting with an anti-NDR phosphopeptide antibody. Intensities of the endogenous NDR1(PThr-444)/NDR2(PThr-442) signals (left hand immunoblot panel) or endogenous NDR1(PThr-444)/NDR2(PThr-442) + FLAG-NDR2(PThr-442) (right hand immunoblot panel) were quantified by scanning densitometry and expressed relative to the values obtained with calyculin A (50 nM, $20 \mathrm{~min})$ for each single experimental grouping. Results are means \pm SEM for 3 (A) or 4 (B) independent preparations of myocytes.

Fig. 6. Stimulation of NDR2 activity in the FLAG-NDR2-PIFtide chimera. Myocytes were infected with an adenovirus (Adv) empty vector, with Adv.FLAG-NDR2(wild-type) or with Adv.FLAG-NDR2-PIFtide. The relative levels of expression of FLAG-NDR2(wild-type) and FLAG-NDR2-PIFtide were assessed with an antibody to the FLAG epitope (upper panel). Myocytes expressing the transgenes were exposed to $200 \mathrm{nM}$ calyculin A for $20 \mathrm{~min}$ and the activities of FLAG-NDR2(wild type) or FLAG-NDR2-PIFtide were measured (lower panel). Results are expressed relative to the FLAG-NDR2(wild-type) infected myocytes exposed to $200 \mathrm{nM}$ calyculin A for $20 \mathrm{~min}$ and are means \pm SEM for 6 independent preparations of myocytes. The results shown were not corrected for expression levels and the Adv blank was not subtracted (but see the Results section for these calculations).

Fig. 7. Subcellular localisation of FLAG-NDR2. Myocytes were infected with Adv.FLAGNDR2 and were stained with (A) monoclonal anti-FLAG, (B) phalloidin, or (C) Hoechst 33258 and a representative image of a single field is shown.

Fig. 8. Phosphorylation of NDR1(Thr-444)/NDR2(Thr-442) in intact hearts. In each separate set of experiments, following a 15 min retrograde stabilisation perfusion, ex vivo rat heart preparations were either (i) perfused retrogradely for a further $20 \mathrm{~min}$, (ii) rendered globallyischemic for $10 \mathrm{~min}$ by occlusion of the aortic inflow, (iii) reperfused retrogradely for $10 \mathrm{~min}$ following global ischaemia (10 min), or (iv) perfused retrogradely with $40 \mathrm{nM}$ calyculin A for 
5 min. Phosphorylation of NDR1(Thr-444)/NDR2(Thr-442) was assessed by immunoblotting with an anti-NDR phosphopeptide antibody, and intensities of the NDR1(PThr444)/NDR2(PThr-442) signals were quantified by scanning densitometry and expressed relative to the values obtained with perfusions with calyculin A ( $40 \mathrm{nM}, 5 \mathrm{~min})$ for each single experimental grouping. Results are means \pm SEM for 8 complete sets of experiments. Statistical significance versus the control perfusions: ${ }^{*}, P<0.0001$ versus control by an unpaired two-tailed Student's $t$ test. 
Figure(s)
Click here to download high resolution image

Fuller et al. Fig. 1
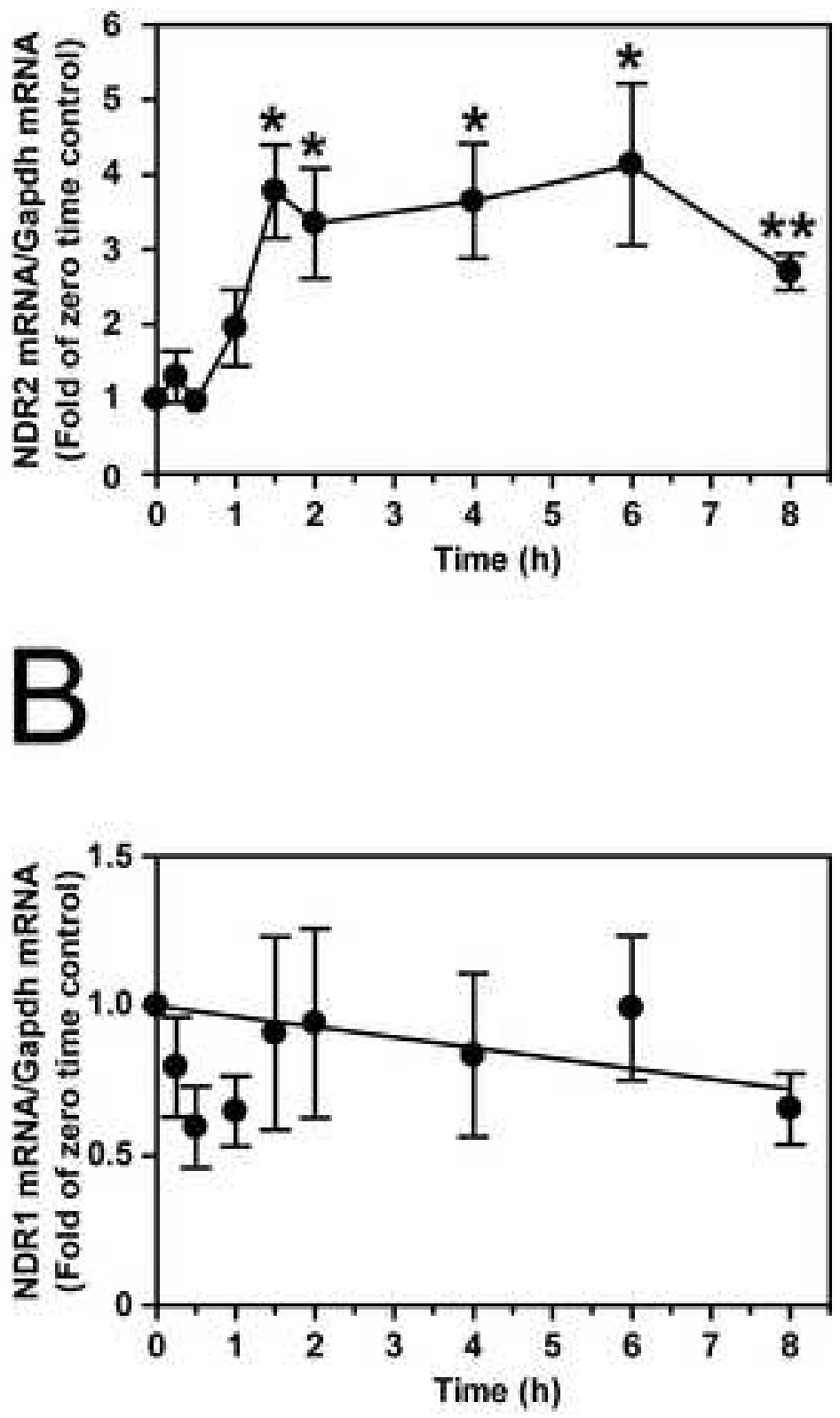


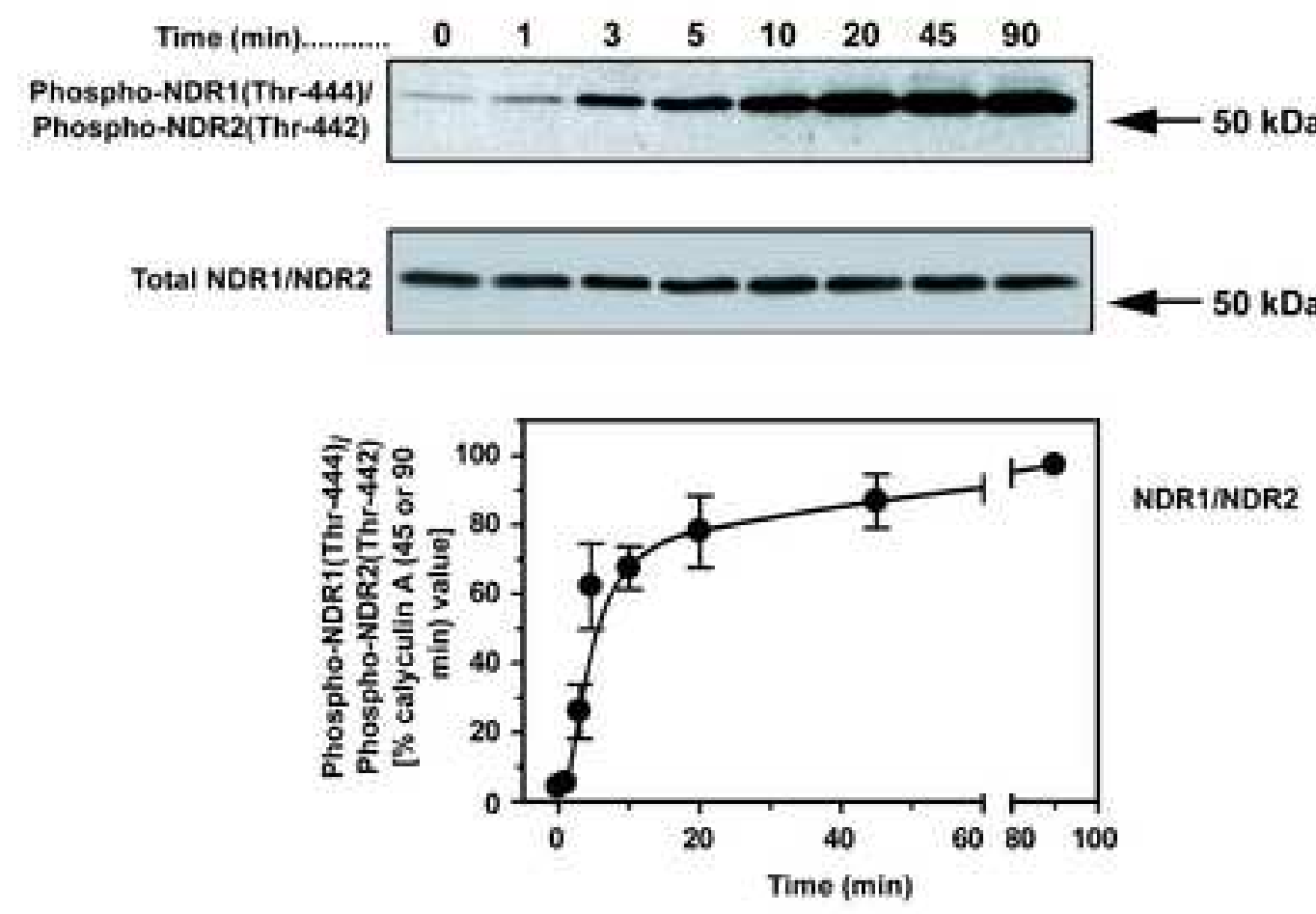

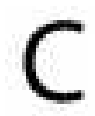

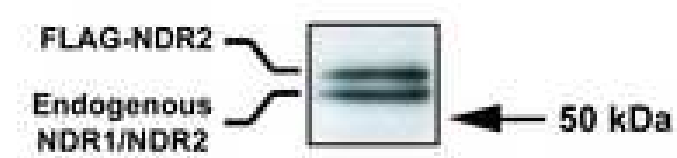

D

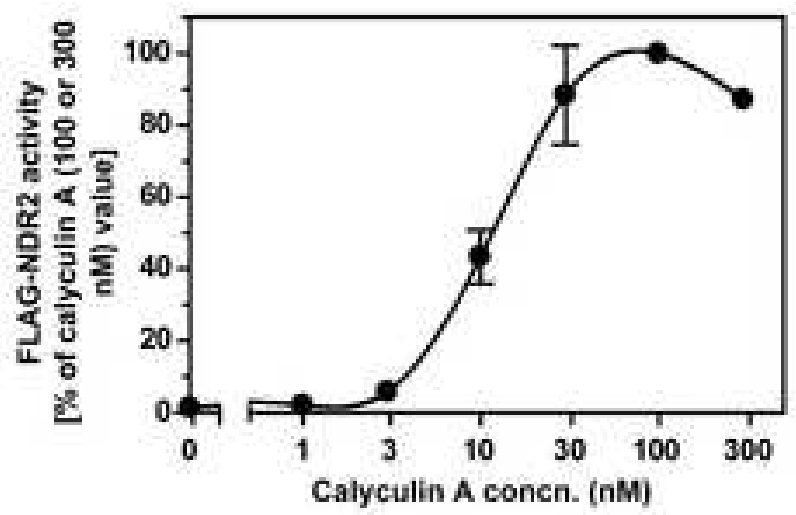

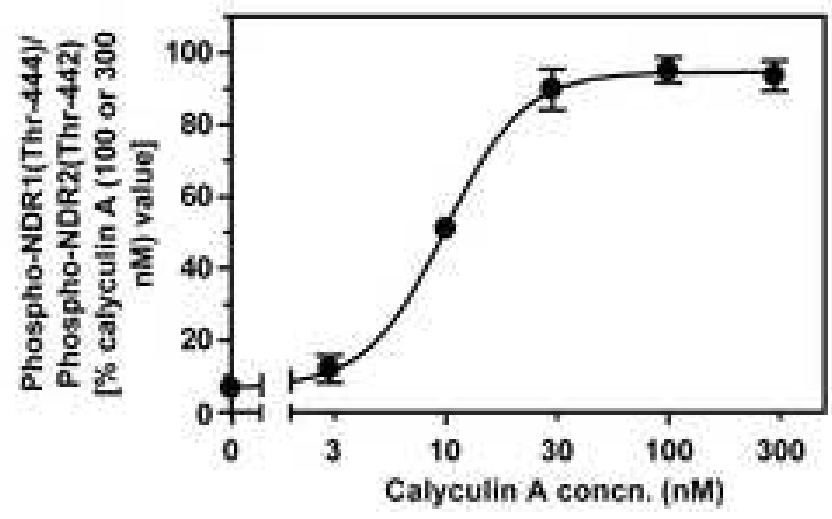


Fuller et al. Fig. 3
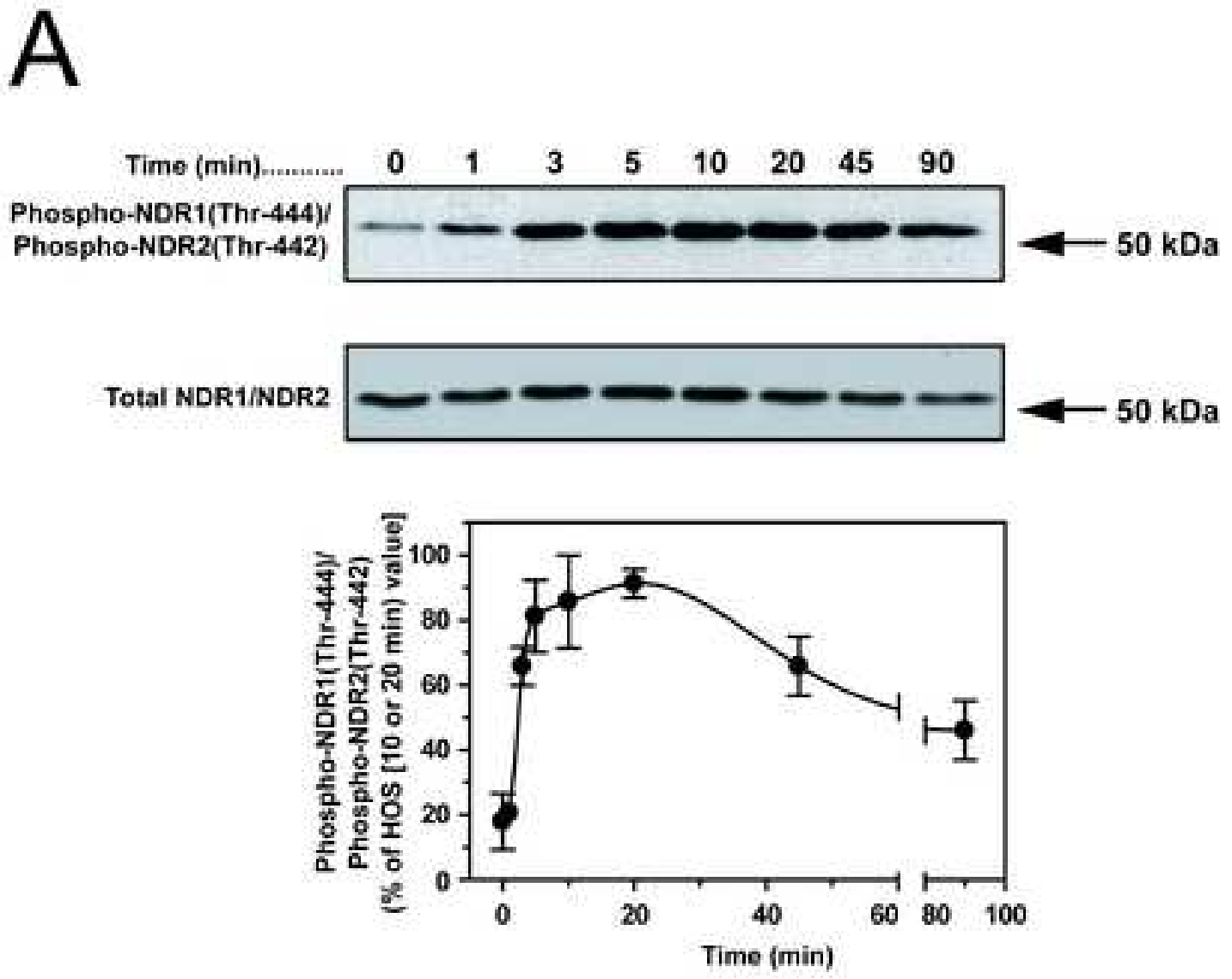

B

Time (min).

$\begin{array}{llllllll}0 & 1 & 3 & 5 & 10 & 20 & 45 & 90\end{array}$

Phospho-NDR1(Thr-444)/

Phospho-NDR2(Thr-442)

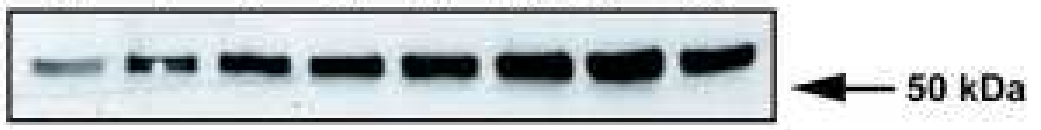

Total NDR1/NDR2
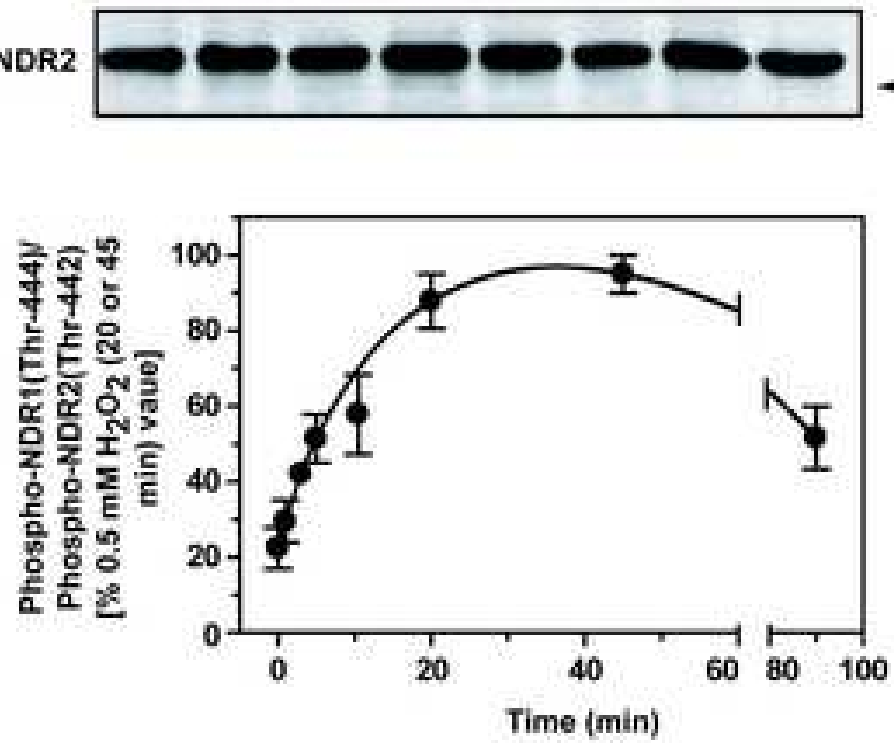
Click here to download high resolution image

Fuller et al. Fig. 3
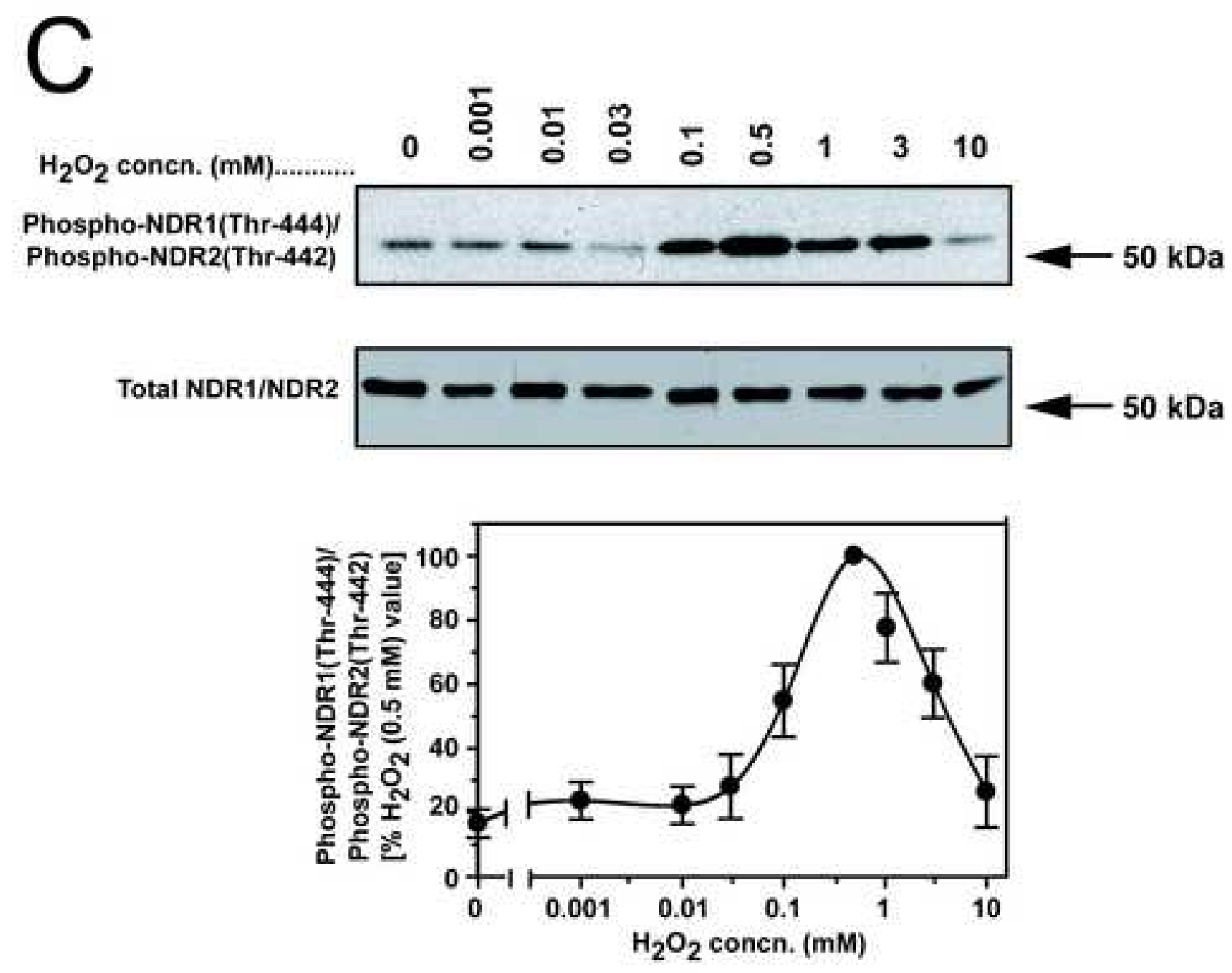
Fuller et al. Fig. 4
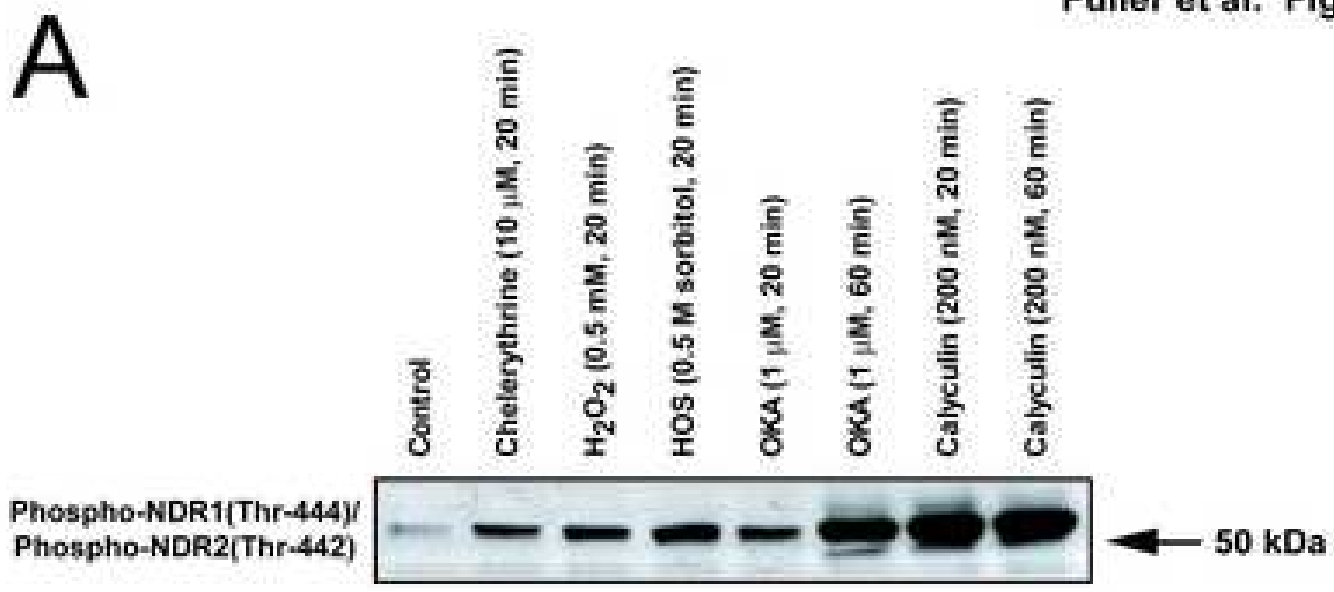

Phospho-NDR1(Thr-444)/

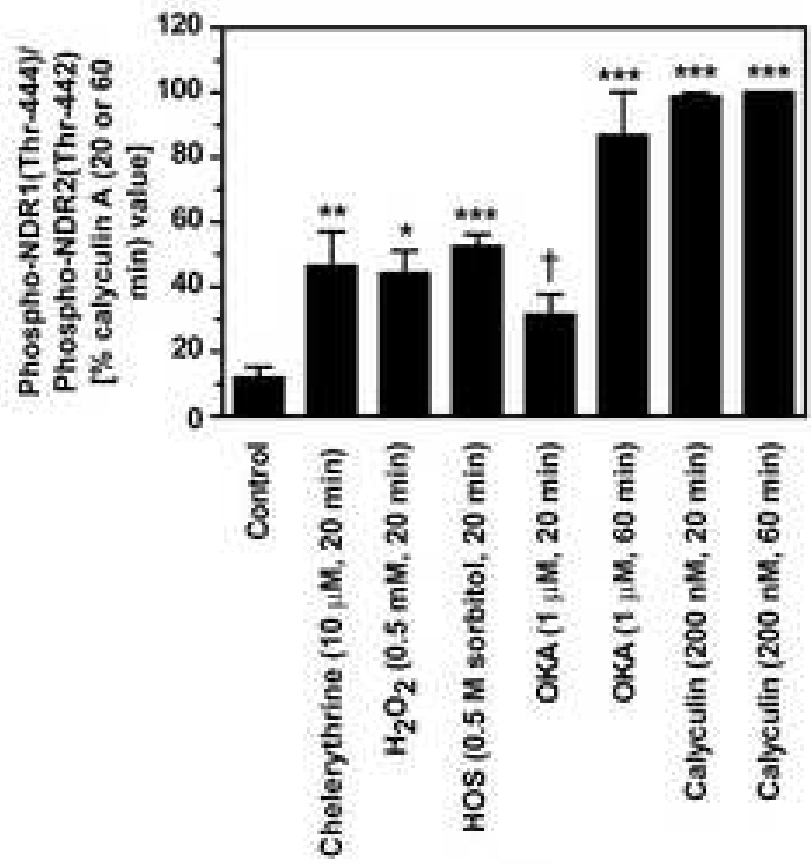

B

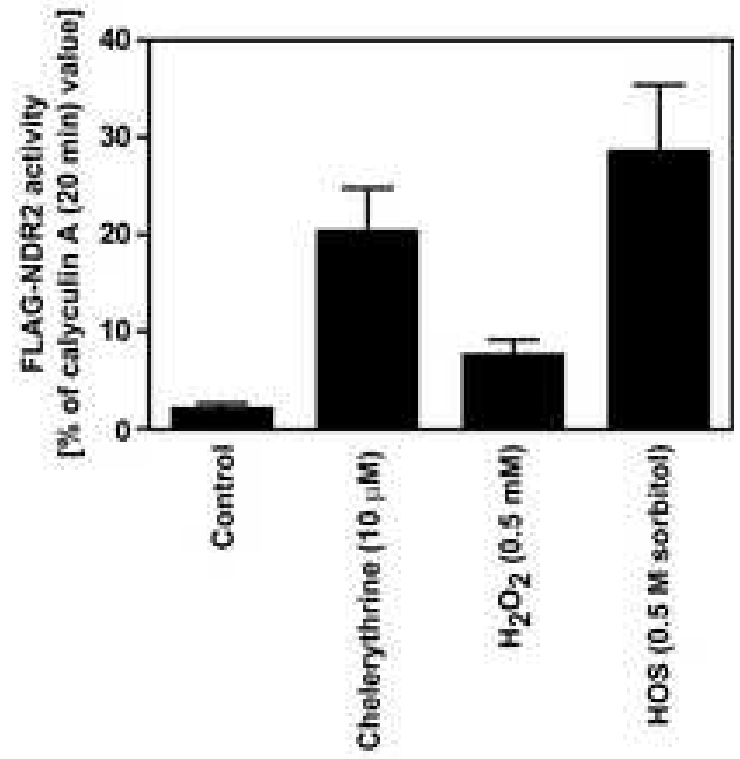

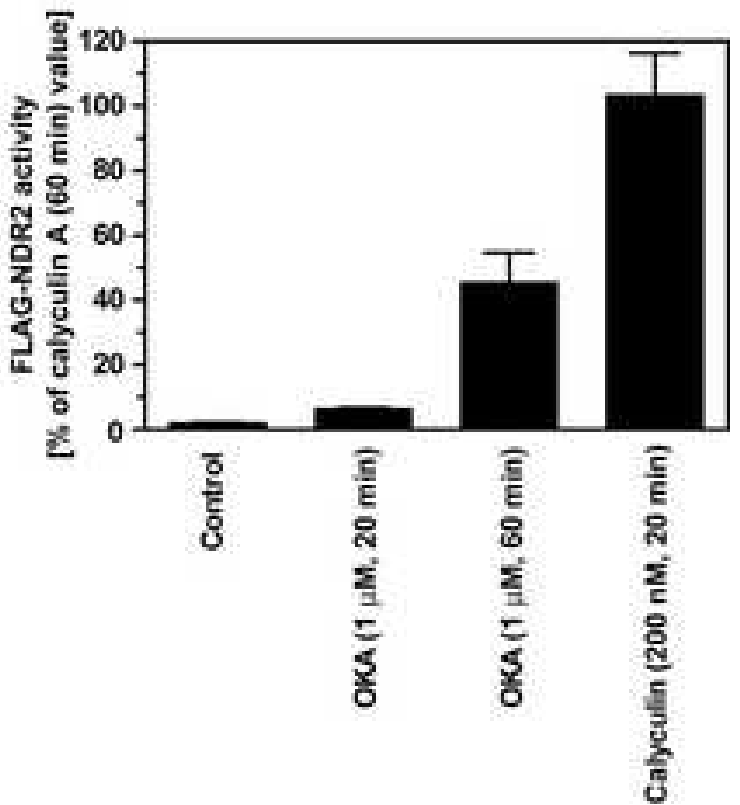


Fuller et al. Fig. 4
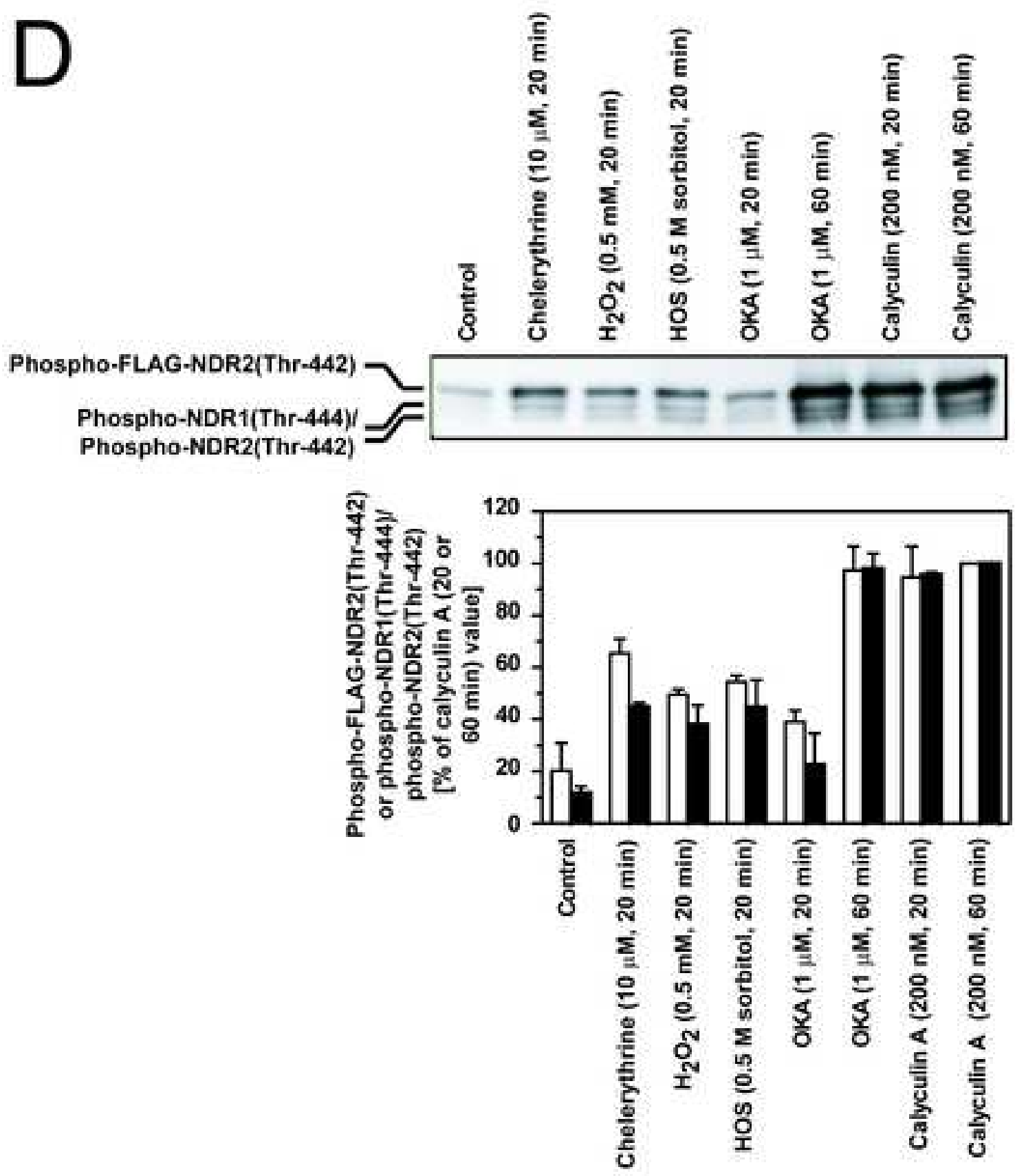

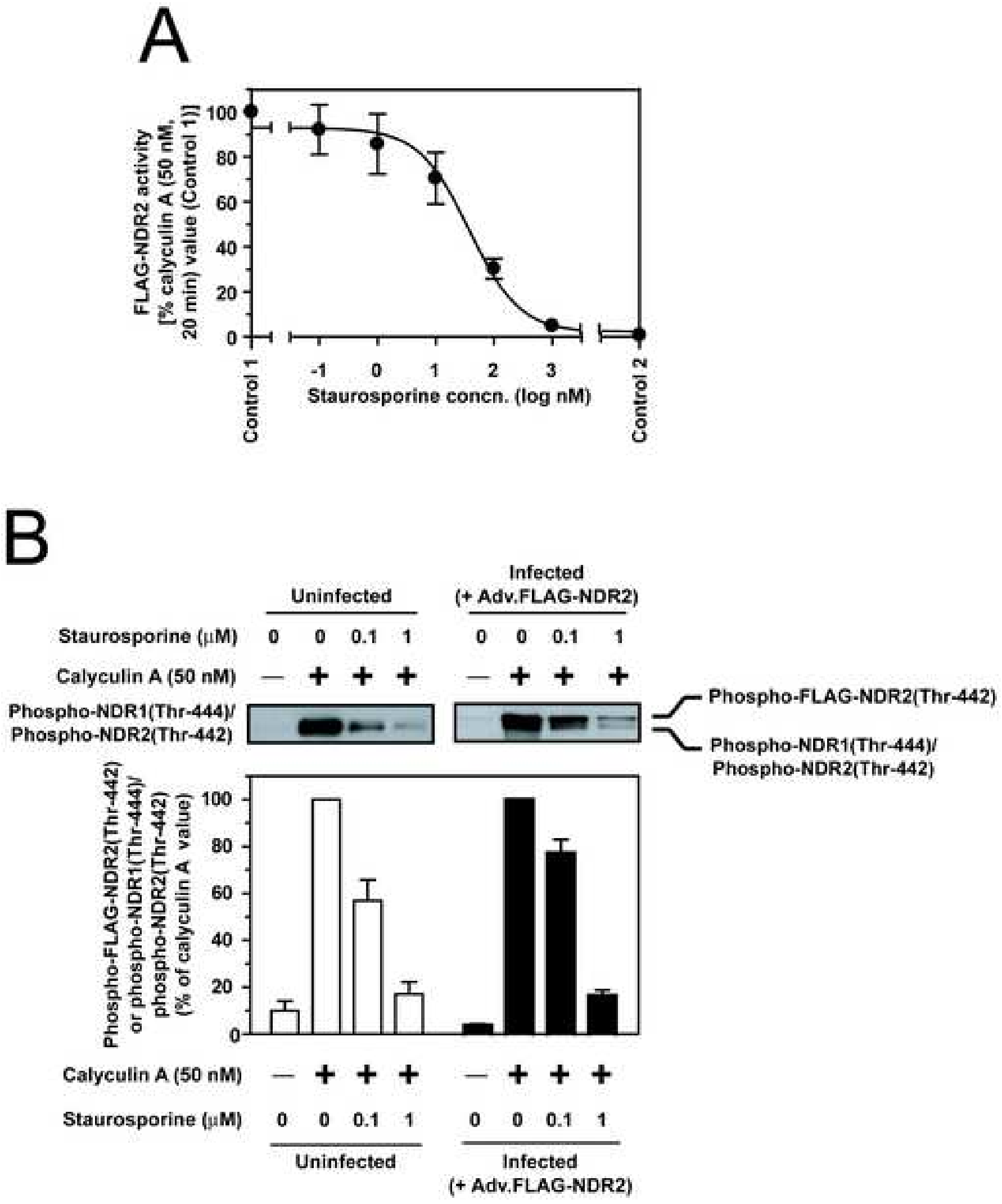
Fuller et al. Fig. 6
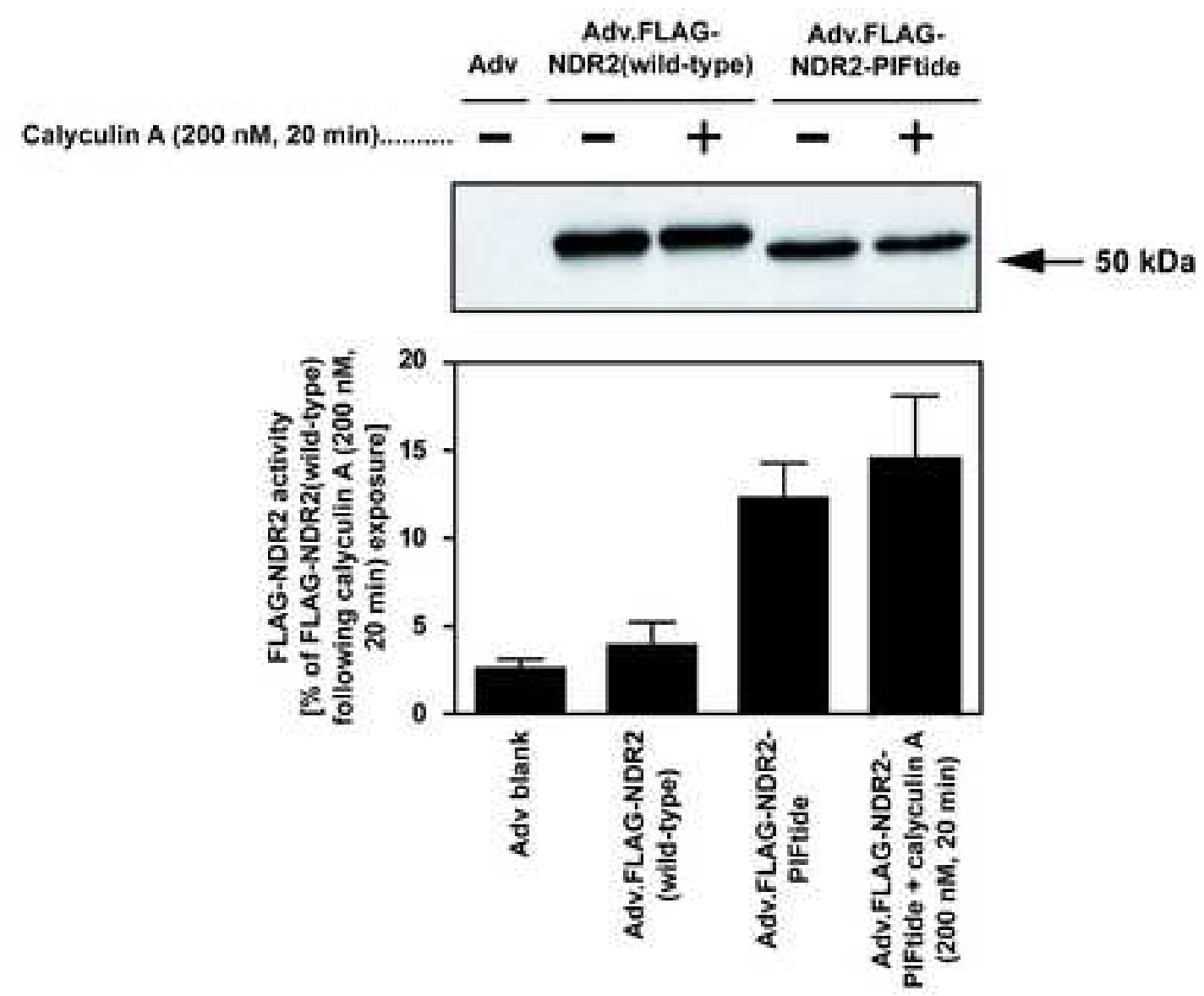

Fuller et al. Fig. 7
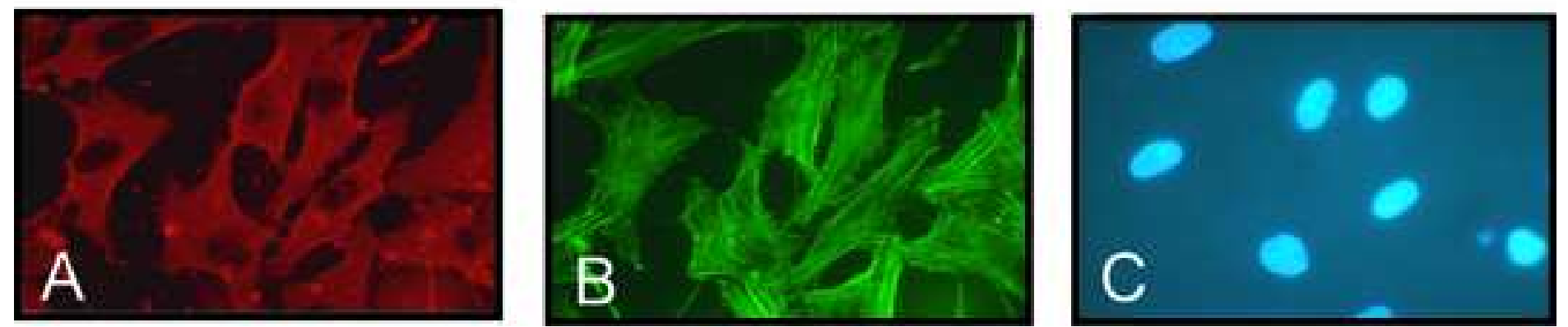
Click here to download high resolution image

Fuller et al. Fig. 8
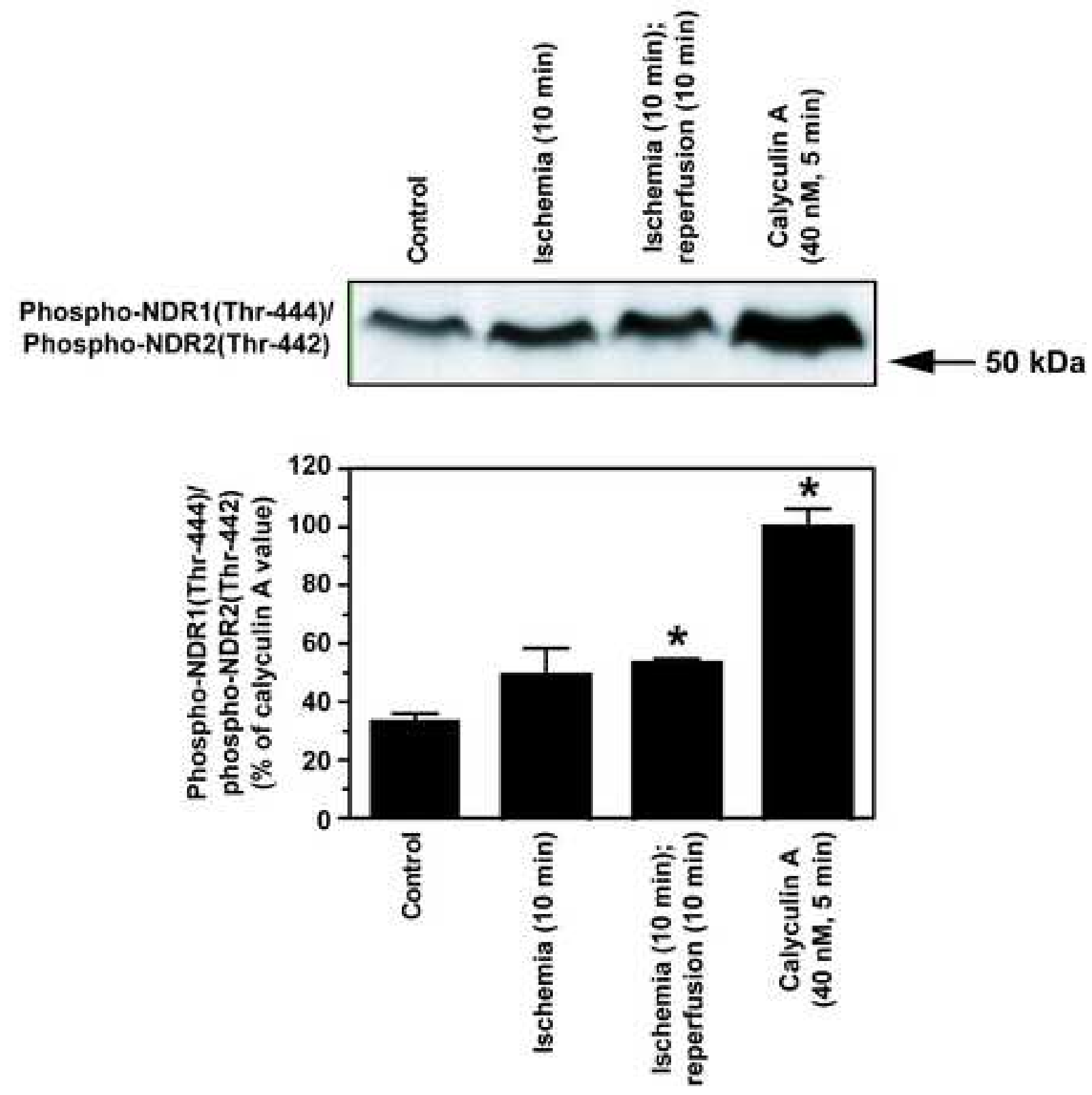


\section{Supplementary material}

\section{Sequencing of rat NDR2 mRNA}

Mouse NDR2 mRNA (Genbank accession no. NM_172734.2) was used to search the rat genome (www.ncbi.nlm.nih.gov/BLAST/) for the orthologous rat NDR2. Because of the incompleteness of the rat genomic sequence, only a partial sequence was identified on rat chromosome 4. Mouse NDR2 mRNA was then used to search for expressed sequence tags (ESTs) allocated to that region of rat chromosome 4 (Genbank accession nos. CV126295.1, CB720056.1, CB611889.1, CO568629.1, CV126546.1, BI290748.1 and CB801811.1). By combining sequences from the rat genome and these ESTs, the nucleotides comprising 1-1258 bp of the open reading frame of rat NDR2 mRNA were obtained. The last 336 bp of mouse NDR2 mRNA open reading frame were used to search against rat genome ESTs, and two additional ESTs were identified (Genbank accession nos. CV126295.1 and CB771034.1). Following this in silico approach, 1-1258bp and 1293-1592bp were obtained for rat NDR2 mRNA. cDNA prepared from neonatal rat ventricular myocytes was used as a template for PCR amplification with sets of primers (spanning predicted exon-exon boundaries) based on the in silico sequence (Supplementary Material, Table 1A). The resulting RT-PCR products were detected by SYBR green staining of agarose gel electrophoretograms and the bands captured under UV illumination. Bands were excised from the gel and purified using QIAQUICK spin columns. The sequence of PCR product with each primer pair was verified by automated sequencing with the indicated (Table 1B) primers (Advanced Biotechnology Centre, Imperial College London) using an ABI 3100 Genetic Analyser. PCR products were sequenced from both ends with the primers originally used for PCR. 


\section{Rapid amplification of cDNA ends (RACE) analysis}

Total RNA was extracted using RNA-Bee from 3 separate myocyte preparations. Quantity and purity of RNAs were assessed from the $A_{260}$ and the $A_{260} / A_{280}$ ratio (1.8-2.0). Total RNA (150 : g) from control or endothelin-1 (ET-1, 100 nM, 2 h) treated myocytes was used for poly(A) ${ }^{+}$RNA purification with oligo dT-cellulose (Biochemica, distributed by Sigma-Aldrich Company Ltd., U.K.). Briefly, total RNA dissolved in deionised water (600 : l) was incubated for 5 min at $65^{\circ} \mathrm{C}$ followed by addition of 1 vol. of $2 \times$ Binding Buffer [1 $\mathrm{M} \mathrm{NaCl}, 20 \mathrm{mM}$ Tris pH 7.5, 2 mM EDTA, 0.1\% (w/v) SDS]. Oligo dT-cellulose (5 mg) in 100 : 1 Binding Buffer was added and mixtures were rotated in room temperature for 15 min. The oligo dT-cellulose was washed twice with Binding Buffer (1 ml) and twice with Washing Buffer [1 ml; 0.2 M NaCl, 10 mM Tris pH 7.5, 1 mM EDTA, $0.05 \%$ (w/v) SDS]. $\operatorname{Poly}(\mathrm{A})^{+}$RNA was eluted from the oligo dT-cellulose by incubation $\left(2 \times 5 \mathrm{~min}, 37^{\circ} \mathrm{C}\right)$ in Elution Buffer [250 : l; 10 mM Tris pH 7.5, 1 mM EDTA, 0.05\% (w/v) SDS]. The purification procedure was repeated and poly $(\mathrm{A})^{+} \mathrm{RNA}$ was precipitated with $4 \mathrm{M} \mathrm{NaCl}$ (50 : l) and two vol. absolute ethanol, followed by centrifugation $\left(10,000 \times \mathrm{g}, 15 \mathrm{~min}, 4^{\circ} \mathrm{C}\right)$. After washing with 70\% (v/v) ethanol and repeated centrifugation, poly(A) ${ }^{+}$RNA was dissolved in deionised water $(8: \mathrm{l})$. Poly $(\mathrm{A})^{+}$RNA was reverse-transcribed using a SMART $^{\mathrm{TM}}$ RACE cDNA Amplification Kit (Clontech). Poly(A) ${ }^{+}$RNA (0.9: g) was mixed with $10 \mathrm{mM}$ oligo-dT primer (1 : l, 5' -CDS primer A or 3'-CDS primer A), $10 \mathrm{mM}$ SMART $^{\mathrm{TM}}$ II A oligonucleotide (1 : l), and deionised water to a total volume of $5: 1$. The reaction mixture was heated $\left(2 \mathrm{~min}, 72^{\circ} \mathrm{C}\right)$ and cooled on ice. Subsequently, $5 \times$ Reaction Buffer (2 : l; 250 mM Tris-HCl, pH 8.3, 30 mM MgCl 2,375 mM KCl), 10 mM dNTP (1 : l ), 20 mM dithiothreitol (DTT, $1:$ l) and PowerScript ${ }^{\mathrm{TM}}$ reverse trancriptase (1: l) were added and mixture was incubated at 42/C for $90 \mathrm{~min}$. Reverse transcriptase was then inactivated 
$\left(72^{\circ} \mathrm{C}, 7 \mathrm{~min}\right)$. RACE cDNA reaction mixture $(10: 1)$ was diluted to $250: 1$ with Tricine-EDTA (10 mM Tricine $\mathrm{pH} 8.5,1 \mathrm{mM}$ EDTA) and stored at $-80^{\circ} \mathrm{C}$. Sequence corresponding to either 5 ' -end or 3 ' -end of NDR2 mRNA were amplified using SMARTTM RACE cDNA Amplification Kit's Universal Primer A Mix (UPM, complementary for the extended 5 '-end of the 5'-RACE cDNA or extended of 3 '-end of 3 '-RACE cDNA after the poly-A tail) and NDR2 gene specific primers. For each PCR reaction, $2.5: 1$ of RACE cDNA was combined with $10 \times$ Advantage ${ }^{\circledR} 2$ Polymerase Buffer [Clontech, 10 : l; 40 mM Tricine-KOH pH 9.2, 15 mM KOAc, 3.5 mM Mg(OAc) $)_{2}$, NDR2 gene specific primer (1 : l; $10: \mathrm{M}), 10 \times \operatorname{UPM}(5: \mathrm{l})$, dNTP $(10 \mathrm{mM})$ and Advantage ${ }^{\circledR} 2$ Polymerase Mix (1 : l). The polymerase chain reaction (PCR) program was as follows: $94^{\circ} \mathrm{C}, 5 \mathrm{~min} ; 5$ cycles of $\left(94^{\circ} \mathrm{C}, 30\right.$ s; $\left.72^{\circ} \mathrm{C}, 2 \mathrm{~min}\right), 5$ cycles of $\left(94^{\circ} \mathrm{C}, 30 \mathrm{~s} ; 70^{\circ} \mathrm{C}, 30 \mathrm{~s} ; 72^{\circ} \mathrm{C}, 2 \mathrm{~min}\right), 25$ cycles $\left(94^{\circ} \mathrm{C}, 30 \mathrm{~s}\right.$; $68^{\circ} \mathrm{C}, 30 \mathrm{~s} ; 72^{\circ} \mathrm{C}, 2 \mathrm{~min}$ ) and $72^{\circ} \mathrm{C}, 4 \mathrm{~min}$. The PCR reaction mixture was diluted $1: 50$ with MilliQ water and the diluted RACE reaction $(2.5: 1)$ was used as a template in nested PCR with appropriate NDR2 primers (Supplementary Material, Table 1C) and the SMART ${ }^{\mathrm{TM}}$ RACE cDNA Amplification Kit Nested UPM primer using a similar PCR protocol as in original RACE PCR. Nested PCR products were analysed on SYBR Green-stained agarose gels and the bands were captured under UV illumination. Products were excised from the gel and purified using QIAQUICK PCR purification columns for automated sequencing (Advanced Biotechnology Centre, Imperial College London) using an ABI 3100 Genetic Analyser. The ClustalW program (www.ebi.ac.uk/clustalw) was used for multiple alignment of sequencing results (Supplementary Material, Fig. 1), and the rat NDR2 mRNA sequence submitted to Genbank (accession no. EF444939.1). 


\section{Adenoviral vectors}

Adenoviral vectors were prepared using the AdEasy ${ }^{\mathrm{TM}}$ XL Adenoviral Vector System (Stratagene). To facilitate identification, separation and immunoprecipitation of virally infected proteins from endogenous NDR2, adenoviral constructs were tagged with a FLAG ${ }^{\circledR}$ sequence (DYKDDDDK) at their N-terminus. A FLAG ${ }^{\circledR}$ sequence was first introduced between the BglII and KpnI restriction sites in the multiple cloning site (MCS) of the pShuttle-CMV vector by synthesis of an oligonucleotide cassette containing the FLAG sequence preceded by an ATG start codon. The complementary oligonucleotides used were: Forward:

5'-GATCTACCATGGACTACAAAGACGATGACGACAAGGGTACCGTCGACGC-3';

Reverse:

5'-GGCCGCGTCGACGGTACCCTTGTCGTCATCGTCTTTGTAGTCCATGGTA-3' with the FLAG $^{\circledR}$ sequence underlined and emboldened and its antisense complement underlined and italicised. The oligonucleotides were annealed and phosphorylated, resulting in a cassette flanked by "cut" BglII and NotI sites, which was then inserted into the corresponding restriction sites in the multiple cloning site of pShuttle-CMV. This strategy, which replaces the KpnI-NotI fragment of pShuttle-CMV with an identical sequence in the cassette insert, was used to avoid the potential difficulty of cutting both the BglII and KpnI sites which lie immediately adjacent to each other in pShuttle-CMV.

The rat NDR2 coding sequence was subsequently amplified from rat cDNA by PCR with Pfu polymerase using forward primer (5'-GACGACAAGGGTACCATGGCAATGACGGCAGGGGC-3') and reverse primer

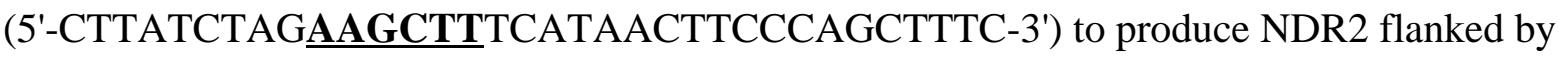


KpnI (forward primer) and HindIII (reverse primer) sites which are underlined and

emboldened. These were then used for insertion into the corresponding sites in the prepared FLAG-pShuttle-CMV, downstream and in frame with the FLAG tag sequence. NDR2PIF was prepared similarly but using reverse primer

\section{5'-CTTATCTAGAAGCTTTCACCAGTCGGCGATGTAGTCGAAGTCGCGGAACATCT} CCTGCTCCTCTTTGTAGTCAGGCTCTGTGGTATTCG-3' which generates a product in which the C-terminal hydrophobic domain of NDR2 (residues 433-464) is replaced with the 3-phosphoinositide-dependent kinase 1 interacting fragment (PIF) of human protein kinase N2 (PKN2, NP_006247 residues 969-983, sequence EQEMFRDFDYVADW), as has been previously performed for human NDR2 [1]. The orthologous sequence in rat PKN2 is identical to human PKN2 except for the substitution of Ile- for Val-980. All constructs were sequenced using an ABI 3100 Genetic Analyser (Advanced Biotechnology Centre, Imperial College London). Shuttle plasmids containing verified sequences were then linearised with PmeI and used to transform BJ5183-AD-1 cells which were subsequently screened for homologous recombination. Adenoviral plasmids from positive recombinants were expanded in XL10Gold cells, linearised with PacI and used to transform HEK293 cells, as described in the AdEasy ${ }^{\mathrm{TM}}$ XL Adenoviral Vector System manual. Viruses were amplified through subsequent re-infection of HEK293 cells.

\section{Reference}

[1] M.R. Stegert, R. Tamaskovic, S. J. Bichsel, A. Hergovich, B. A. Hemmings, J. Biol. Chem. 279 (2004) 23806. 
SUPPLEMENTARY MATERIAL TABLE 1. Primer sequences.

A. Sequences for RT-PCR of NDR2 mRNA

\begin{tabular}{llll}
\hline Primer & \multicolumn{1}{c}{ Sequence $\left(5^{\prime} \rightarrow 3^{\prime}\right)$} & $\begin{array}{c}\text { Position in NDR2 } \\
(\text { EF444939.1) }\end{array}$ & \multicolumn{1}{c}{ Primer pairing } \\
& & & \\
\hline F1 & TGGCAATGACGGCAGGGGCAAC & $152 \rightarrow 173$ & F1R1, F1R2 \\
R1 & TCCTCGTCTGCCAGTCCTTC & $316 \leftarrow 336$ & F1R1 \\
R2 & TTCTCCTCGTCTGCCAGTCC & $319 \leftarrow 338$ & F1R2 \\
F2 & ACTGTGGCCAAGCTCACGCTG & $214 \rightarrow 234$ & F2R3 \\
R3 & TGGCATAGATGTGCCCTGTG & $483 \leftarrow 502$ & F2R3 \\
F3 & TGTGGCCAAGCTCACGCTGG & $216 \rightarrow 235$ & F3R4 \\
R4 & TCATGGCATAGATGTGCCCT & $486 \leftarrow 505$ & F3R4 \\
F4 & AGAAGGACTGGCAGACGAGG & $315 \rightarrow 334$ & F4R5 \\
R5 & CTGAGATGTAGAACTGCGTC & $717 \leftarrow 736$ & F4R5 \\
F5, & CAGGTGGCTCATATCCGAGC & $541 \rightarrow 560$ & F5R6, F5R7, F5R8 \\
R6 & AGCCTAGCTGGTGGATTGCGTC & $754 \leftarrow 775$ & F5R6 \\
F7 & ACACCAGACTACATTGCTCCAGAGG & $1006 \rightarrow 1030$ & F5R7 \\
R8 & AAGTCCTTGGCTTTCTCAGAGACG & $1197 \leftarrow 1220$ & F5R8 \\
F8 & TGGCATTTCCTCCAGAGGTGC, & $1175 \rightarrow 1195$ & F8R9, F8R10 \\
R9 & TGGAGCCTCGCTGGGTCAGC & $1494 \leftarrow 1513$ & F8R9, F8R10 \\
F9 & AGGTCATCCGTTCTTTGAGGGTGT & $1284 \rightarrow 1308$ & F9R10 \\
R10 & ACGTGGGGATGGAGCCTCGCTG & $1501 \leftarrow 1522$ & F8R10, F9R10 \\
& & & \\
\hline
\end{tabular}


B. Primers for NDR2 sequencing

\begin{tabular}{lll}
\hline Primer & \multicolumn{1}{c}{ Sequence $\left(5^{\prime} \rightarrow 3^{\prime}\right)$} & $\begin{array}{c}\text { Position in } \\
\text { NDR2 } \\
(\text { EF444939.1) }\end{array}$ \\
\hline F14 & AGGTCATCCGTTCTTTGAGGGTG & $1284 \rightarrow 1306$ \\
R15 & AGGTTCTACAGGCAGCTTTCATGG & $2379 \rightarrow 2356$ \\
F15 & TCCGGAGCATAGATGACACCTCA & $1355 \rightarrow 1377$ \\
R14 & ACGCACTAGGTAGGTTCTACAGGCAG & $2365 \leftarrow 2390$ \\
R15 & AGGTTCTACAGGCAGCTTTCATGG & $2356 \rightarrow 2379$ \\
F16 & CACATCGCACTGATGGCACC & $1896 \rightarrow 1915$ \\
& & \\
\hline
\end{tabular}

C. Primer sequences for NDR2 RACE

\begin{tabular}{llcl}
\hline Primer & \multicolumn{1}{c}{ Sequence $\left(5^{\prime} \rightarrow 3^{\prime}\right)$} & $\begin{array}{c}\text { Position in } \\
\text { NDR2 } \\
\text { (EF444939.1) }\end{array}$ & Function \\
& & & \\
\hline & & & \\
F10 & ACGCAATCCACCAGCTAGGCTTCATCCACCGGGA & $755 \rightarrow 788$ & $3^{\prime}$-RACE \\
F11 & AGACGCTGGCATTCCTCCAGAGGTGCCCGT & $1169 \rightarrow 1199$ & $3^{\prime}$-RACE, Nested PCR for F10 product \\
F12 & GGTTTGAAGGGCTGACCCAGCGAGGCTCCATCC & $1484 \rightarrow 1516$ & $3^{\prime}$-RACE, Nested PCR for F11 product \\
R11 & TGGACCAGCCGCACCTCTCCGAAAGCTCCTC & $443 \leftarrow 473$ & $5^{\prime}$-RACE, Nested PCR for R12 product \\
R12 & TCCCGGTGGATGAAGCCTAGCTGGTGGATTGCGTC & $754 \leftarrow 788$ & $5^{\prime}$-RACE, Nested PCR for R13 product \\
R13 & AGACGGGCACCTCTGGAGGAAATGCCAGCGTC & $1170 \leftarrow 1201$ & $5^{\prime}$-RACE \\
\hline
\end{tabular}


SUPPLEMENTARY MATERIAL TABLE 2. Agonists and interventions ineffective in causing phosphorylation of endogenous NDR1/NDR2 or activation of FLAG-NDR2.

A. Agonists and interventions tested which did not increase phosphorylation of NDR1(Thr444)/NDR2(Thr-442).

Endothelin-1 (100 nM, vasoactive peptide, pro-hypertrophic agonist in cardiac myocytes)

Tumor necrosis factor " (10 ng/ml, pro-inflammatory cytokine)

Interleukin $1 \$(10 \mathrm{ng} / \mathrm{ml}$, pro-inflammatory cytokine)

Leukemia inhibitory factor (10 nU/ml, gp130-linked cytokine receptor agonist)

Cardiotrophin-1 (1 nM, gp130-linked cytokine receptor agonist, pro-hypertrophic agonist in cardiac myocytes)

Ionomycin $(1: \mathrm{M})$

A23187 (20 : M, Ca ${ }^{2+}$ ionophore)

Anisomycin (50 ng/ml, protein synthesis inhibitor, cytotoxic stressor)

Cycloheximide (20 : M, protein synthesis inhibitor, cytotoxic stressor)

Staurosporine (1 : M, generalized Ser-/Thr-protein kinase, pro-apoptotic in cardiac myocytes)

Cyclosporin A [1 : M, phosphoprotein phosphatase 3 (calcineurin) inhibitor]

Doxorubicin (5: M, antineoplastic anthracycline, pro-apoptotic in cardiac myocytes and cardiomyopathic)

Insulin (300 nM, receptor protein tyrosine kinase agonist polypeptide)

Platelet-derived growth factor (20 ng/ml, receptor protein tyrosine kinase agonist polypeptide)

Epidermal growth factor (20 ng/ml, receptor protein tyrosine kinase agonist polypeptide)

Phorbol 12-myristate 13-acetate (1 : M, activator of classical and novel isoforms of protein kinase C, pro-hypertrophic agonist in cardiac myocytes)

Phenylephrine (100 : M, "-adrenergic receptor agonist, pro-hypertrophic agonist in cardiac myocytes) 
B. Agonists and agents tested which did not increase the activity of FLAG-tagged NDR2.

Na arsenite (0.5 mM, cytotoxic stressor)

Cycloheximide (20 : M, protein synthesis inhibitor, cytotoxic stressor)

Isoproterenol (10 : M, \$-adrenergic receptor agonist, positive inotrope, lusitrope and chronotrope)

Phenylephrine (10 : M, " -adrenergic receptor agonist, pro-hypertrophic agonist in cardiac myocytes)

Norepinephrine (10: M, " -/\$-adrenergic receptor agonist, pro-hypertrophic agonist in cardiac myocytes)

Endothelin-1 (100 nM, vasoactive peptide, pro-hypertrophic agonist in cardiac myocytes)

$\mathrm{KCl}$ (40 mM, cardioplegic intervention)

pH6.6 or pH 7.8 (pH lowered from or raised above control value of 7.4)

Lipopolysaccharides from Escherichia coli 0111.B4 or from Salmonella typhimurium serotype minnesota (500 ng/ml, endotoxin/cytotoxic stressors)

Lipopolysaccharides (rough strains) from Salmonella typhimurium serotype minnesota Re 595 (500 ng/ml, endotoxin/cytotoxic stressor) 
SUPPLEMENTARY MATERIAL FIG. 1. Alignments are shown for the amino acid sequences encoded by the rat NDR2 (rNDR2, EF444939.1) cDNA, human NDR1 (hNDR1, NP_009202.1), mouse NDR1 (mNDR1, NP_598876.1), the predicted sequence for rNDR1 (genomic contig Rn20_WGA2119_4), hNDR2 (NP_055815.1) and mNDR2 (NP_766322.1). The peptide sequences to which the hNDR1 antibody was raised (59-86) and to which the hNDR1(PThr-444) antibody was raised (436-450) are emboldened and underlined. 
rNDR2 mNDR2 hNDR2 rNDR I mNDR 1 hNDR 1

rNDR2 mNDR2 hNDR2 rNDR 1 mNDR I hNDR 1

rNDR2 mNDR2 hNDR2 rNDR 1 mNDR I hNDR 1

rNDR2 mNDR2 hNDR2 rNDR 1 mNDR 1 hNDR1

rNDR2 mNDR2 hNDR2 rNDR 1 mNDR 1 hNDR1

rNDR2 mNDR2 hNDR2 rNDR 1 mNDR I hNDR1

rNDR2 mNDR2 hNDR2 rNDR 1 mNDR 1 hNDR1

rNDR2 mNDR2 hNDR2 rNDR 1 MNDR 1 hNDRI

MAMTAGATTT FPMSSHTRER VTVAKLTLEN FYSNLILQHE ERETRQKKLE VAMEEEGLAD 60 MAMTAGATTT FPMSNHTRER VTVAKLTLEN FYSNLILQHE ERETRQKKLE VAMEEEGLAD 60 MAMTAGTTTT FPMSNHTRER VTVAKLTLEN FYSNLILQHE ERETRQKKLE VAMEEEGLAD 60 MAMT-GSTPC SSMSNHTKER VTMTKVTLEN FYSNLIAQHE EREMRQKKLE KVMEEEGLKD 59 MAMT-GSTPC SSMSNHTKER VTMTKVTLEN FYSNLIAQHE EREMRQKKLE KVMEEEGLKD 59 MAMT-GSTPC SSMSNHTKER VTMTKVTLEN FYSNLIAQHE EREMRQKKLE KVMEEEGLKD 59

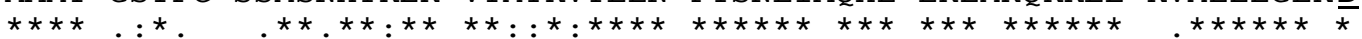

EEKKLRRSOH ARKETEFLRL KRTRLGLDDF ESLKVIGRGA FGEVRLVOKK DTGHIYAMKI 120 EEKKLRRSQH ARKETEFLRL KRTRLGLDDF ESLKVIGRGA FGEVRLVQKK DTGHIYAMKI 120 EEKKLRRSQH ARKETEFLRL KRTRLGLDDF ESLKVIGRGA FGEVRLVQKK DTGHIYAMKI 120 EEKRLRRSAH ARKETEFLRL KRTRLGLEDF ESLKVIGRGA FGEVRLVQKK DTGHVYAMKI 119 EEKRLRRSAH ARKETEFLRL KRTRLGLEDF ESLKVIGRGA FGEVRLVQKK DTGHVYAMKI 119 EEKRLRRSAH ARKETEFLRL KRTRLGLEDF ESLKVIGRGA FGEVRLVQKK DTGHVYAMKI 119

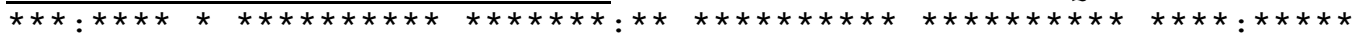

LRKADMLEKE OVAHIRAERD ILVEADGAWV VKMFYSFODK RNLYLIMEFL PGGDMMTLLM 180 LRKADMLEKE QVAHIRAERD ILVEADGAWV VKMFYSFQDK RNLYLIMEFL PGGDMMTLLM 180 LRKSDMLEKE QVAHIRAERD ILVEADGAWV VKMFYSFQDK RNLYLIMEFL PGGDMMTLLM 180 LRKADMLEKE QVGHIRAERD ILVEADSLWV VRMFYSFQDK LNLYLIMEFL PGGDMMTLLM 179 LRKADMLEKE QVGHIRAERD ILVEADSLWV VKMFYSFQDK LNLYLIMEFL PGGDMMTLLM 179 LRKADMLEKE QVGHIRAERD ILVEADSLWV VKMFYSFQDK LNLYLIMEFL PGGDMMTLLM 179

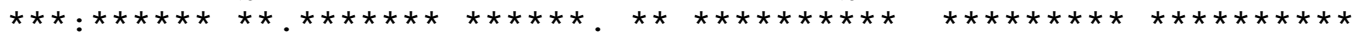

KKDTLTEEET OFYISETVLA IDAIHOLGFI HRDIKPDNLL LDAKGHVKLS DFGLCTGLKK 240 KKDTLTEEET QFYISETVLA IDAIHQLGFI HRDVKPDNLL LDAKGHVKLS DFGLCTGLKK 240 KKDTLTEEET QFYISETVLA IDAIHQLGFI HRDIKPDNLL LDAKGHVKLS DFGLCTGLKK 240 KKDTLTEEET QFYIAETVLA IDSIHQLGFI HRDIKPDNLL LDSKGHVKLS DFGLCTGLKK 239 KKDTLTEEET QFYIAETVLA IDSIHQLGFI HRDIKPDNLL LDSKGHVKLS DFGLCTGLKK 239 KKDTLTEEET QFYIAETVLA IDSIHQLGFI HRDIKPDNLL LDSKGHVKLS DFGLCTGLKK 239

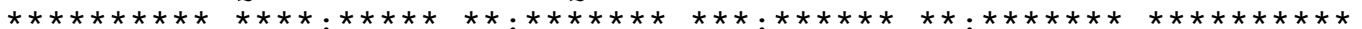

AHRTEFYRNL THNPPSDFSF QNMNSKRKAE TWKKNRRQLA YSTVGTPDYI APEVFMQTGY 300 AHRTEFYRNL THNPPSDFSF QNMNSKRKAE TWKKNRRQLA YSTVGTPDYI APEVFMQTGY 300 AHRTEFYRNL THNPPSDFSF QNMNSKRKAE TWKKNRRQLA YSTVGTPDYI APEVFMQTGY 300 AHRTEFYRNL NHSLPSDFTF QNMNSKRKAE TWKRNRRQLA FSTVGTPDYI APEVFMQTGY 299 AHRTEFYRNL NHSLPSDFTF QNMNSKRKAE TWKRNRRQLA FSTVGTPDYI APEVFMQTGY 299 AHRTEFYRNL NHSLPSDFTF QNMNSKRKAE TWKRNRRQLA FSTVGTPDYI APEVFMQTGY 299

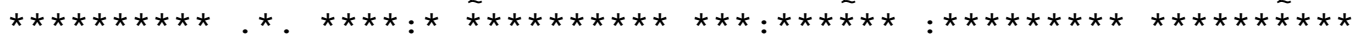

NKLCDWWSLG VIMYEMLIGF PPFCSETPQE TYRKVMSWKE TLAFPPEVPV SEKAKDLILR 360 NKLCDWWSLG VIMYEMLIGF PPFCSETPQE TYRKVMSWKE TLAFPPEVPV SEKAKDLILR 360 NKLCDWWSLG VIMYEMLIGY PPFCSETPQE TYRKVMNWKE TLVFPPEVPI SEKAKDLILR 360 NKLCDWWSLG VIMYEMLIGY PPFCSETPQE TYKKVMNWKE TLTFPPEVPV SEKAKGLILR 359 NKLCDWWSLG VIMYEMLIGY PPFCSETPQE TYKKVMNWKE TLTFPPEVPV SEKAKGLILR 359 NKLCDWWSLG VIMYEMLIGY PPFCSETPQE TYKKVMNWKE TLTFPPEVPI SEKAKDLILR 359

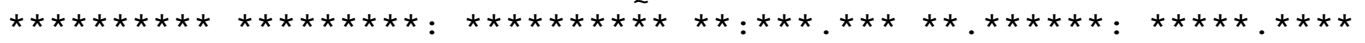

FCTDSENRIG NGGVEEIKGH PFFEGVDWGH IRERPAAIPI EIRSIDDTSN FDDFPESDIL 420 FCTDSENRIG NGGVEEIKGH PFFEGVDWGH IRERPAAIPI EIRSIDDTSN FDDFPESDIL 420 FCIDSENRIG NSGVEEIKGH PFFEGVDWEH IRERPAAIPI EIKSIDDTSN FDDFPESDIL 420 FCCEWEHRIG APGVEEIKSN PFFEGVDWEH IRERPAAISI EIKSIDDTSN FDEFPESDIL 419 FCCEWEHRIG APGVEEIKNN LFFEGVDWEH IRERPAAISI EIKSIDDTSN FDEFPESDIL 419 FCCEWEHRIG APGVEEIKSN SFFEGVDWEH IRERPAAISI EIKSIDDTSN FDEFPESDIL 419

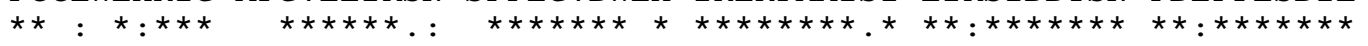

QPVPNTT--- EPDYKSKDWV FLNYTYKRFE GLTQRGSIPT YMKAGKL 464 QPVPNTT-- - EPDYKSKDWV FLNYTYKRFE GLTQRGSIPT YMKAGKL 464 QPVPNTT--- EPDYKSKDWV FLNYTYKRFE GLTQRGSIPT YMKAGKL 464 KPTVTTSNHP ETDYKSKDWV FINYTYKRFE GLTARGAIPS YMKAAK 465 KPTVTTSSHP ETDYKNKDWV FINYTYKRFE GLTARGAIPS YMKAAK 465 KPTVATSNHP ETDYKNKDWV FINYTYKRFE GLTARGAIPS YMKAAK 465

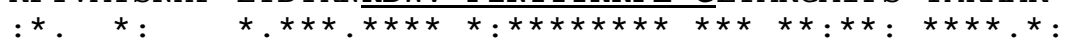

